\title{
Systematic Review \\ Effect of Probiotics on Psychiatric Symptoms and Central Nervous System Functions in Human Health and Disease: A Systematic Review and Meta-Analysis
}

\author{
Charlotte Le Morvan de Sequeira, Charlotte Hengstberger, Paul Enck (D) and Isabelle Mack*
}

\section{check for}

updates

Citation: Le Morvan de Sequeira, C.; Hengstberger, C.; Enck, P.; Mack, I. Effect of Probiotics on Psychiatric Symptoms and Central Nervous System Functions in Human Health and Disease: A Systematic Review and Meta-Analysis. Nutrients 2022, 14, 621. https://doi.org/10.3390/ nu14030621

Academic Editors: Laura Soldati and Luigi Barrea

Received: 15 December 2021

Accepted: 25 January 2022

Published: 30 January 2022

Publisher's Note: MDPI stays neutral with regard to jurisdictional claims in published maps and institutional affiliations.

Copyright: (C) 2022 by the authors. Licensee MDPI, Basel, Switzerland. This article is an open access article distributed under the terms and conditions of the Creative Commons Attribution (CC BY) license (https:// creativecommons.org/licenses/by/ $4.0 /)$.
Department of Psychosomatic Medicine and Psychotherapy, University Medical Hospital, 72076 Tübingen, Germany; charlotte.le-morvan-de-sequeira@student.uni-tuebingen.de (C.L.M.d.S.); charlotte.hengstberger@student.uni-tuebingen.de (C.H.); paul.enck@uni-tuebingen.de (P.E.)

* Correspondence: isabelle.mack@uni-tuebingen.de

\begin{abstract}
Background: The gut microbiota impacts on central nervous system (CNS) function via the microbiota-gut-brain axis. Thus, therapeutics targeting the gut microbiota such as probiotics have the potential for improving mental health. This meta-analysis synthesizes the evidence regarding the impacts of probiotics on psychological well-being, psychiatric symptoms and CNS functioning. Methods: The Preferred Reporting Items for Systematic Reviews and Meta-Analyses guidelines were applied for executing this review using the databases PubMed, Web of Science and Cochrane Library. The data were summarized at qualitative and quantitative level. Results: Fifty-four randomized placebo-controlled studies were included, of which 30 were eligible for meta-analysis. If investigated, the probiotics mostly exerted effects on CNS function. Most probiotics did not affect mood, stress, anxiety, depression and psychiatric distress when compared to placebo at the qualitative level. At quantitative level, depression and psychiatric distress improved slightly in the probiotic condition (depression: mean difference -0.37 (95\% CI: $-0.55,-0.20) ; p \leq 0.0001$; psychiatric distress: mean difference $-0.33(95 \% \mathrm{CI}:-0.53,-0.13) ; p=0.001)$. Conclusions: To date it is unclear to which extent and in which specific areas next generation probiotics selected and developed for their ability to improve psychiatric condition and potentially other CNS functions are promising.
\end{abstract}

Keywords: probiotics; paraprobiotics; central nervous system (CNS); anxiety; depression; stress; mood; psychiatric distress; systematic review; meta-analysis

\section{Introduction}

Mental health disorders such as anxiety and depression are highly prevalent and are steadily increasing. Their impact on quality of life of the patients and their families and their economic burden for society is enormous [1]. In addition, anxiety and depression are often comorbidities of other mental and somatic disorders, especially in chronic conditions [2]. Since the gastrointestinal (GI) microbiota influences brain function and behavior, understanding the mechanisms could provide resources for improving psychological well-being in health and maybe even in psychiatric conditions [3].

The GI microbiota is the total amount of living microorganisms colonizing the GI tract of a host organism [4]. The gut is exposed to numerous potential pathogens. Thus, it is necessary for the host to prevent their uncontrolled penetration into the body. Immune defense via unspecific mechanisms along with the gut-associated lymphoid tissue are essential in this interplay. Furthermore, the immune-modulating potential of the GI microbiota [5] and some probiotics has been reported [6]. However, the interactions between the indigenous microorganisms and the host are mostly beneficial. The former are important for the maintenance of the gut barrier function but also the overall health of the host $[7,8]$. Interestingly, the composition of the gut microbiota is different in patients with severe and moderate major depression disorder in comparison to healthy individuals [5]. In line with 
this, the gut microbiota can exert effects on central nervous system (CNS) functions via the microbiota-gut-brain axis, which has been extensively reviewed elsewhere [9-11].

Thus, it is logical, why therapeutics targeting the GI microbiota, such as prebiotics, probiotics and postbiotics / paraprobiotics (inactivated bacteria or fractions) [12,13] and fermented milks [14], are of potential interest for (i) influencing mood and stress resilience in health and disease and, (ii) adjunct treatment of psychiatric and functional CNS disorders [9]. In pre-clinical studies with rodents, the evidence for probiotics being beneficial for memory performance, improving stress response, and even reducing anxiety and depression, is convincing. However, not all pre-clinical studies report superiority of probiotics over placebo, the literature being reviewed elsewhere $[9,15,16]$. Besides probiotics, there are also other potential GI microbiota-modulating substances [17].

In humans, the situation is far less clear, not only because studies in rodents outnumber human studies, but also because a number of experiments can only be conducted in pre-clinical models due to ethical reasons and translation into human models is extremely challenging and not always possible. Beyond this, the lack in the field becomes obvious when considering that only six randomized controlled trials (RCT) [18-23] exist investigating the effects of probiotics on CNS function by brain imaging studies. Nevertheless, the field has been growing over the last decade and the questions arise whether or not probiotics and postbiotics are able to influence psychological well-being, psychiatric symptoms and central functions and, if yes, to which extent effects can be expected in humans.

Thus, the aim of this systematic review and meta-analysis was to synthesize the evidence regarding the effects of probiotics and postbiotics on (i) psychological well-being (mood and stress response), (ii) psychiatric symptoms (depression, anxiety, psychiatric distress) and (iii) central functioning. The latter is defined as neural brain activity measured by electroencephalography (EEG) and by imaging methods in both healthy participants and patients with functional CNS or psychiatric disorders.

\section{Materials and Methods}

\subsection{Literature Information Sources and Search Strategy}

The Preferred Reporting Items for Systematic Reviews and Meta-Analyses (PRISMA) guidelines were applied for executing this review [24]. The 3 databases Web of Science, PubMed, and Cochrane Library were searched on 20 March 2021 and updated on 24 September 2021. The strategy of the full search is reported in the Supporting Information Text S1, consisting of the two modules, i.e., probiotics and CNS function. This review is registered on the PROSPERO platform (CRD42021253080).

\subsection{Eligibility Criteria}

The five PICOS dimensions, i.e., participants (P), interventions (I), comparators (C), outcome $(\mathrm{O})$ and study design (S) [25] were applied to define the eligibility criteria.

Participants: Participants were adults of all ages and both sexes, either healthy or with a specified functional CNS or psychiatric disorder: attention-deficit hyperactivity disorders (ADHD), autism spectrum disorders (ASD), bipolar disorders (BD), depression, tension headache (TD), neurotic, stress-associated and somatoform disorders and affective disorders. Structural and neurodegenerative disorders, including multiple sclerosis, Alzheimer's, Huntington's, ALS, and Parkinson's, conditions secondary to infections, conditions with proven (or suspected) genetic or other somatic origin and conditions with hormonal origins like pregnancy were excluded. This also excludes CNS diseases with a traumatic, vascular, metabolic, autoimmune and neoplastic origin. Somatoform disorders were excluded unless a psychologic component is a prerequisite for diagnosis; this restricts fibromyalgia syndrome (FMS) and chronic fatigue syndrome (CFS) to the subgroup with proven psychiatric comorbidity. Functional bowel disorders were excluded and reviewed elsewhere [26].

Interventions: Trials assessing the effectivity of viable and non-viable microorganisms or microbial cell extracts to be used as probiotics, paraprobiotics, bacterial lysate, psy- 
chobiotics, single- and/or multi-strain preparations. The minimum of treatment duration had to be 3 weeks. Exclusion criteria were studies investigating prebiotics, synbiotics or antibiotics.

Comparators: A blinded placebo control group was necessary for inclusion.

Outcome Measures: Behavioral measures, neuropsychological measures (psychometric tests) and neurophysiological measures (e.g., electroencephalography, magnetoencephalography and functional magnetic resonance imaging). In the qualitative analysis, studies that only involved neuroendocrine, neurochemical and neuroimmunologic measures (laboratory) were not included and only mentioned in the discussion (e.g., measuring neurochemical level, HPA axis activity). The reason is the heterogeneity of studies tested and their methodological differences.

Study design: Randomized, double-blinded, placebo-controlled trials.

\subsection{Study Selection, Data Collection and Organization}

First, the results of the database searches were combined. Next, the duplicates were removed followed by screening the titles and abstracts. Finally, the evaluation of the fulltext articles for eligibility was performed (CLMdS and IM). Uncertainties were discussed between the authors (CLMdS, IM) $(<5 \%)$. Discrepancies were solved by including a third person (PE). For classification, the studies were assigned to one of six groups.

1. Group 1-Probiotics and Depression

2. Group 2-Probiotics and Anxiety

3. Group 3-Probiotics and Stress

4. Group 4-Probiotics and Cognitive Function

5. Group 5-Probiotics and Mental Health and Mood

6. Group 6-Probiotics and other CNS states

\subsection{Data Items and Statistics}

For study characterization, the information on year of publication, country of origin, study type, probiotic intervention, method for data collection, study outcomes including behavioral or neuropsychological validated questionnaires, sample characteristics (sample size, age, sex) was extracted from each included study. Across the studies, the characteristics are reported as frequency and per cent (\%) or median (interquartile range), minimum and maximum for sample size, intake time, sex and age.

The evaluation of data was performed qualitatively and also quantitatively (metaanalysis) where possible.

The qualitative analyses synthesized the findings for their direction of change between the groups. This was necessary, because (i) some studies reported insufficient data (e.g., studies only mentioned that no group differences existed without reporting the data) and/or (ii) the heterogeneity of the applied measurements was too high for a quantitative summary $[27,28]$.

A random-effect model for parallel-group designed studies was applied for metaanalysis, using the software package Review Manager, version 5.4 [29]. The data of the questionnaires are presented as mean and SD separately for the control and intervention groups. The difference is expressed as standardized mean difference and $95 \%$ confidence interval and visualized as forest plots. Statistical heterogeneity was explored by visual inspection of forest plots and using the $\mathrm{I}^{2}$ statistics to quantify inconsistency between the studies. Subgroup analyses were conducted for intake period (4-8 weeks versus 9-24 weeks intake time), the type of probiotic preparation (single versus multi-strain probiotics), the location of the study (Asia vs Not Asia), the application form (liquid/powder versus capsule/pills) and the health state (healthy vs diagnosed psychiatric disorder) for reducing heterogeneity and/or to advance the understanding of the findings. Participants were considered as healthy if they had not been diagnosed with a psychiatric disorder according to validated guidelines such as the Diagnostic and Statistical Manual of Mental Disorders (DSM). 
Several studies used more than one questionnaire to assess the same outcome. For example, to assess the symptoms of anxiety, the State Trait Anxiety Inventory (STAI), the Beck Anxiety Inventory (BAI) and Hamilton Anxiety Rating Scale (HAM-A) were used in parallel in one study [30]. To compare the data across different questionnaires, in case of multiple questionnaires, for anxiety the STAI state was used and for depression the Beck Depression Inventory (BDI). The reason is that these were the most utilized questionnaires for the respective outcomes. However, in addition, all data are also reported questionnaire-wise (in the Supplementary Materials) but without total summary for methodological correctness.

Missing data were requested from authors and $46.15 \%$ (6 out of 13 ) responded to the inquiry.

\subsection{Risk of Bias}

The risk of bias was assessed using the Cochrane risk-of-bias tool for randomized trials (RoB 2) [31]. It is structured into five domains addressing different types of bias: randomization process, deviations from the intended interventions, missing outcome data, measurement of the outcome and selection of the reported result. First, specific questions in each domain need to be answered for each single study. Next, the RoB2 algorithm calculates the risks of the individual domains. Finally, an overall risk is computed and expressed as "low risk of bias", "risk with some concerns" or "high risk of bias".

\section{Results}

The literature search process is presented in Figure 1. The search identified 427 studies of which 54 remained for analysis.

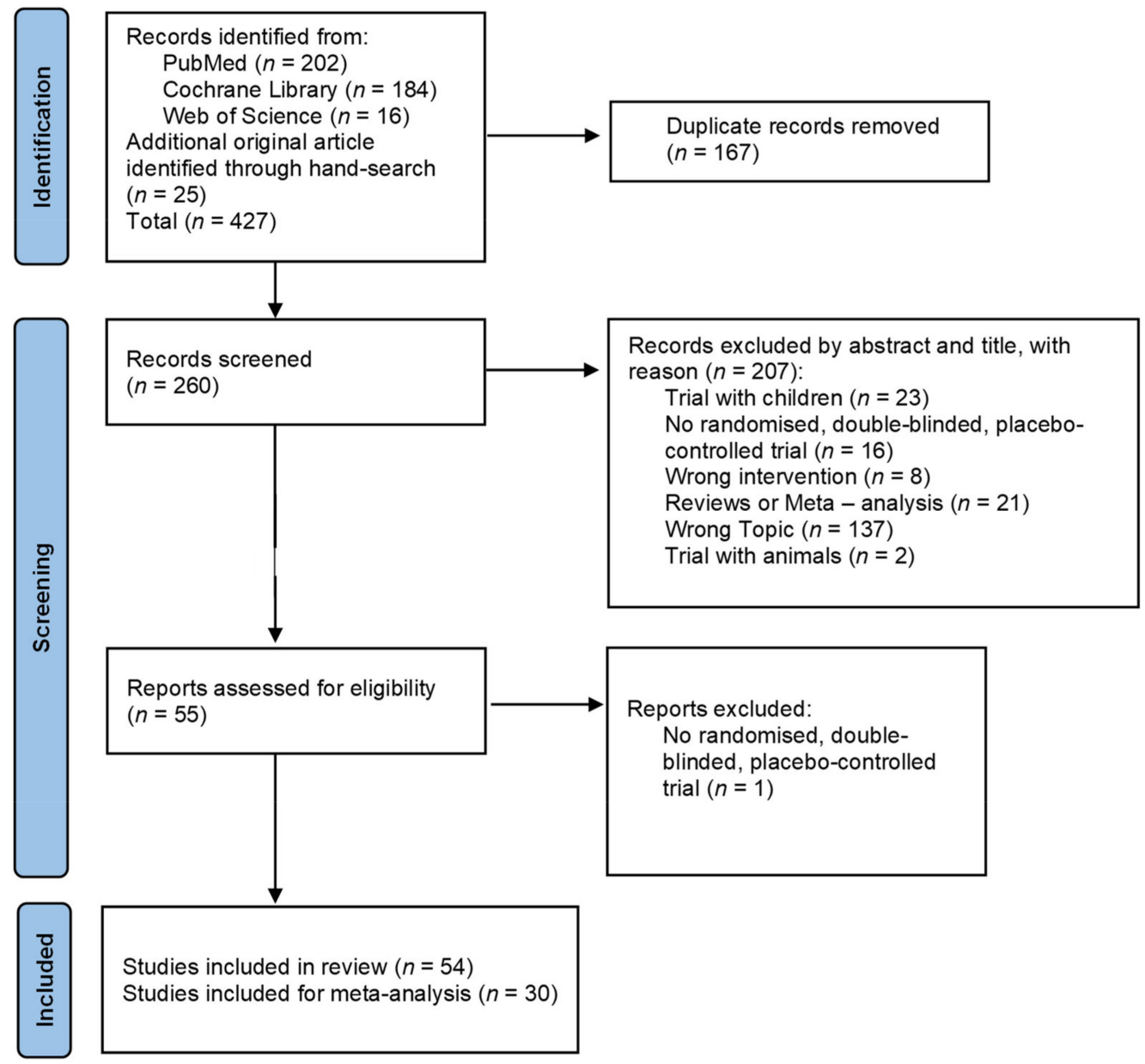

Figure 1. PRISMA flow chart for study inclusion. 


\subsection{Study Selection and Categorization}

The characteristics of the single trials are reported in Table 1 along with the detailed information on bacterial species of the probiotics provide in Table S1 and an overview of the applied questionnaires and tests and their abbreviations in Table S2. The characteristics across the studies are given below and in Table S3.

\subsection{Summary of Study Characteristics}

The publishing dates of the studies were between 2007 and 2021. Most studies were conducted in Asia $(n=28 ; 51.9 \%)[30,32,33,36,41,43-45,47,48,50,51,53-58,62,63,69,70,72,74,75,77-79]$, followed by Europe $(n=18 ; 33.3 \%)$ [18-20,22,34,35,37,40,42,46,49,52,59,60,64,65,67,73], America $(n=7 ; 12 \%)[21,38,39,61,68,71,76]$ and Oceania $(n=1 ; 1.9 \%)$ [66]. In total, the 54 trials included 4449 randomized participants; 3700 participants finished trials and 4130 participants were analyzed. The median age was $35.6(24.9-50.1)$ years and $60.1 \%$ were women. The intervention durations ranged between 3 and 24 weeks with a median length of $8(4-12)$ weeks. Most probiotics were ingested as powders $(n=19 ; 35.2 \%)$ followed by liquids such as milk $(n=12 ; 22.2 \%)$, capsules $(n=13 ; 24.1 \%)$ and applications with tablets or pills $(n=7 ; 13 \%)$. Three studies did not report the application form $(5.6 \%)$.

The intake ranged from one to four applications of probiotics per day. Mostly, probiotic intake was once per day $(n=46 ; 85.2 \%)$. The studies investigated single-strain $(n=29 ; 53.7 \%)$ and multi-strain probiotics $(n=25 ; 46.3 \%)$. The latter consisted of two to twenty different probiotic strains. The median number of colony-forming units (CFUs) was $1 \times 10^{10}\left(2.5 \times 10^{9}-2.1 \times 10^{10}\right)$ CFUs per day. The range was between $2.3 \times 10^{7}$ and $1.4 \times 10^{11} \mathrm{CFUs}$ per day. Five studies did not report the CFU $(9.3 \%)$

Most trials compared a probiotic with a placebo group. Twelve $(22.2 \%)$ studies investigated more than two groups. Four studies compared the placebo group with several probiotic groups with different CFU concentration [36,54,72,76], while two studies compared them with different species $[46,48]$. The study with the most probiotic groups investigated five different groups [76]. Three studies compared the results of the probiotic groups with the results of a placebo group and also with the results of a no interventional group $[18,19,21]$. Five studies were conducted as cross-over design and all other studies as parallel group design $[34,37,49,70,78]$.

Several studies added to their probiotic treatment other substances such as vitamin D $(n=3 ; 5.6 \%)[41,62,64]$, selective serotonin reuptake inhibitors (SSRI) $(n=2 ; 3.7 \%)[30,67]$, selenium [63] or $\alpha$-lactalbumin [69]. While Benton et al. (2007) never mentioned any division of the participants in groups [35], another study, Mohammadi et al. (2016), used combinations with capsules and yoghurts with and without probiotics [53].

Only four studies examined the impact on CNS function by using functional magnetic resonance imaging [18-21], while only six studies used electroencephalography (EEG) $[33,34,43,49,56,75]$. There was also one study applying magnetoencephalography (MEG) [22], another using actigraphic measurements [78] and one study with electrodermal responses (EDR) [33]. The other studies investigated anxiety, depression, stress, psychiatric distress and other mental or behavioral states by validated questionnaires. An overview of the results of the most frequently used questionnaires are presented studyand questionnaire-wise in Table S4.

Overall, 41 studies presented data of healthy participants with no diagnosed psychiatric disorder $(75.9 \%)$ and 13 studies (24.1\%) of participants with diagnosed psychiatric disorders according to DSM, such as major depressive disorder $(n=2 ; 3.7 \%)[32,67]$, depression $(n=2 ; 3.7 \%)[48,79]$ and schizophrenia $(n=3 ; 5.6 \%)[38,41,71]$. In addition, studies also included participants with mild cognitive impairment [44], bipolar I/schizoaffective disorder in a manic episode [39], general anxiety disorder [30], chronic fatigue syndrome [61], chronic primary insomnia [43] and fibromyalgia [65]. In addition, five studies only included participants when they achieved a pathological score in questionnaires (9.3\%). 
Table 1. Trial characteristics of single studies. A summary of the raw data as direction of change of the applied questionnaires are presented. It is questionnaire specific whether or not a decrease implies improvement or deterioration and accordingly, whether or not an increase implies improvement or deterioration Psychiatric symptoms and CNS changes in the probiotic versus the placebo group: $\leftrightarrow$ : no statistical differences between the groups; $\downarrow$ : significant reduction $p<0.05$; $\downarrow \downarrow$ : significant reduction $p<0.01$; $\downarrow \downarrow \downarrow$ : significant reduction $p<0.001 ; \uparrow$ : significant increase $p<0.05$; $\uparrow \uparrow$ : significant increase $p<0.01$; A: analyzed; $\alpha$ LA: $\alpha$-Lactalbumin; AUT: Austria; CAN: Canada; CFU: Colony forming unit; CHN: China; CN: Control group; CTG: Combined treatment group; DEU: Deutschland; DSM-5: American Diagnostic and Statistical Manual of Mental Disorders (5th Edition); DTG: Dietary treatment group (hypocaloric diet); ESP: Spain; FMS: Fibromyalgia; FRA: France; IRL: Ireland; IRN: Iran; ITA: Italy; IU: International Unit; JPN: Japan; KOR: Korea; LHFM: Lactobacillus helveticus-fermented milk; LP: Lactobacillus paracasei MCC1849; MCI: Mild cognitive impairment; MDD: Major depressive disorder; MYS: Malaysia; N: Number; NI: No intervention group; NLD: Netherlands; NR: Not reported; NWL: Normal weight lean; NWO: Normal weight obese; NZL: New Zealand; PL: Placebo group; POL: Poland; PR: Probiotic group; PRE: Prebiotic group; PreobOB: Pre-obese/obese; RCT: Randomized controlled trial; SEM: Standard error of mean; SE: Standard error; SD: Standard deviation; SSRI: Selective Serotonin Reuptake Inhibitor; SWE: Sweden; TWN: Taiwan; VAS: Visual Analog Scale. More explanations are provided in Table S2.

\begin{tabular}{|c|c|c|c|c|c|c|}
\hline Author (Year) & Country & Subject & $\begin{array}{l}\text { Intake } \\
\text { Length (Week) }\end{array}$ & $\begin{array}{l}\text { SampleSize (A); Sex (f\%); AgeMean } \\
\text { (SD); HealthCondition; Groups (N) }\end{array}$ & $\begin{array}{l}\text { Probiotic Species (N); Dose; Times } \\
\text { Intake per Day; Application }\end{array}$ & Outcomes \\
\hline Akkasheh et al. (2016) [32] & IRN & Depression & 8 & $\begin{array}{l}40 \text { ( } 40) ; \text { f: } 85 \% \text {; age: PL } \rightarrow 36.2 \text { (8.2), } \\
\text { PR } \rightarrow 38.3 \text { (12.1); MDD; groups: PL (20), } \\
\text { PR (20) }\end{array}$ & $\begin{array}{l}\text { Tak Gen Zist Pharmaceutical Company } \\
\text { (3); each } 2 \times 10^{9} \mathrm{CFU} / \mathrm{g} ; 1 / \text { day; capsule }\end{array}$ & Behavioral: BDI $\downarrow$ \\
\hline Adikari et al. (2020) [33] & MYS & Anxiety & 8 & $\begin{array}{l}20 \text { (19); f: } 0 \% \text {; age: PL } \rightarrow 19 \pm 0.66 \\
\text { PR } \rightarrow 19 \pm 0.81 \text {; healthy; groups: PL (9), } \\
\text { PR (10) }\end{array}$ & $\begin{array}{l}\text { Lactobacillus casei Shirota }(\mathbf{1}) ; 3 \times 10^{10} \\
\text { CFU; } 1 \text { /day; liquid }\end{array}$ & $\begin{array}{l}\text { Neuropsychological: DVT reaction time } \downarrow \text {, } \\
\text { accuracy percentage } \leftrightarrow \\
\text { Neurophysiological: EEG } \leftrightarrow \text { (week } 8 \text { ), } \uparrow \text { theta } \\
\text { brain wave }+ \text { delta brain wave for probiotic group } \\
\text { (week 4); EDR } \leftrightarrow\end{array}$ \\
\hline Bagga et al. (2018) [19] & AUT & $\begin{array}{l}\text { Emotional } \\
\text { brain } \\
\text { signatures }\end{array}$ & 4 & $\begin{array}{l}45 \text { (NR); f: } 48.9 \% \text {; age: } \mathrm{PL} \rightarrow 27.25 \text { (5.78), } \\
\text { PR } \rightarrow 28.27(4.2), \mathrm{NI} \rightarrow 26.87(4.97) ; \\
\text { healthy; groups: PL (15), PR (15), NI (15) }\end{array}$ & $\begin{array}{l}\text { Ecologic } 825 \text { by Winclove Probiotics (9); } \\
7.5 \times 10^{9} \mathrm{CFU} / \mathrm{g} ; 1 \text { /day; powder }\end{array}$ & $\begin{array}{l}\text { Behavioral: Post-hoc comparisons: PANAS } \uparrow \uparrow ; \\
\text { SCL-90 } \leftrightarrow \text {; ADS } \leftrightarrow \text {; LEIDS: Subscales hopelessness } \\
\downarrow \downarrow \text { and risk aversion } \downarrow ; \text { PRP with less decision } \\
\text { change for unpleasant stimuli } \uparrow \uparrow \text { RAU (response } \\
\text { accuracy for unpleasant stimuli) } \\
\text { Neurophysiological: fMRI BOLD contrast } \\
\text { correlated with PANAS positive score } \uparrow ; \text { Emotional } \\
\text { decision-making task (neutral > baseline) contrast: } \\
\text { significant differences (p value NR) in brain } \\
\text { activity in left anterior cingulum compared to NI }\end{array}$ \\
\hline
\end{tabular}


Table 1. Cont.

\begin{tabular}{|c|c|c|c|c|c|c|}
\hline Author (Year) & Country & Subject & $\begin{array}{l}\text { Intake } \\
\text { Length (Week) }\end{array}$ & $\begin{array}{l}\text { SampleSize (A); Sex (f\%); AgeMean } \\
\text { (SD); HealthCondition; Groups (N) }\end{array}$ & $\begin{array}{l}\text { Probiotic Species (N); Dose; Times } \\
\text { Intake per Day; Application }\end{array}$ & Outcomes \\
\hline Bagga et al. (2019) [18] & AUT & $\begin{array}{l}\text { Functional } \\
\text { connectiv- } \\
\text { ity in } \\
\text { brain }\end{array}$ & 4 & $\begin{array}{l}45 \text { (NR); f: } 51.1 \% \text {; age: } 26.24(4.76) \text {; } \\
\text { healthy; groups: PL (15), PR (15), } \\
\text { CN (15) }\end{array}$ & $\begin{array}{l}\text { Ecologic } 825 \text { by Winclove Probiotics (9); } \\
7.5 \times 10^{6} \mathrm{CFU} / \mathrm{g} ; 1 / \text { day; powder }\end{array}$ & $\begin{array}{l}\text { Neurophysiological: significant changes in the } \\
\text { functional connectivity (FC) comparing PRP with } \\
\text { CON and PLP } \\
\text { comparing to CON: } \\
\uparrow \text { Salience network in Cingulate gyrus + } \\
\text { Precuneus cortex), } \\
\downarrow \text { Middle and superior frontal gyrus network in } \\
\text { Frontal pole + Frontal medial cortex; } \\
\text { comparing to PLP: } \\
\downarrow \text { Visual network in Postcentral gyrus + Precuneus } \\
\text { and Default mode network in Frontal pole, } \\
\text { Superior frontal gyrus + Paracingulate gyrus } \\
\text { probiotic intervention did not influence the } \\
\text { structural connectivity }\end{array}$ \\
\hline Benton et al. (2007) [35] & UK & $\begin{array}{l}\text { Mood and } \\
\text { Cognition }\end{array}$ & 3 & $\begin{array}{l}132 \text { (126); f: } 59.52 \% \text {; age: } 61.8 \pm 7.3 \text {; } \\
\text { healthy; groups: NR }\end{array}$ & $\begin{array}{l}\text { Lactobacillus casei Shirota (1); } 6.5 \times 10^{9} \\
\text { CFU; } 1 / \text { day; liquid }\end{array}$ & $\begin{array}{l}\text { Behavioral: POMS: } \leftrightarrow \\
\text { Neuropsychological: Memory: Wechsler Memory } \\
\text { scale, Retrieval from long-term memory, Test of } \\
\text { verbal fluency: } \leftrightarrow \text {; } \\
\text { NART: converted to Z scores: } \leftrightarrow\end{array}$ \\
\hline De Lorenzo et al. (2017) [37] & ITA & $\begin{array}{l}\text { Psychological } \\
\text { Profile }\end{array}$ & 3 & $\begin{array}{l}48 \text { (48); f: } 100 \% \text {; age: NWL30.18 } \pm 2.04 \\
\text { NWO: } 40.00 \pm 12.54, \text { PreobOB: } 33.57 \pm \\
\text { 10.57; healthy; groups: PL (24), PR (24); } \\
\text { RCT Crossover: } 3 \text { weeks intervention, } 3 \\
\text { weeks wash-out, } 3 \text { weeks other } \\
\text { intervention }\end{array}$ & $\begin{array}{l}\text { Biocult Strong, HOMEOSYN (9); each } \\
1.5 \times 10^{10} \mathrm{CFU} / 3 \mathrm{~g} ; 1 / \text { day; powder }\end{array}$ & Behavioral: SCL90R: $\leftrightarrow$; BUT $\downarrow$ (S) \\
\hline Dickerson et al. (2014) [38] & USA & Schizophrenia & 14 & $\begin{array}{l}65 \text { (65); f: } 35.4 \% \text {; age: } \mathrm{PL} \rightarrow 48.1 \text { (9.4), } \\
\text { PR } \rightarrow 44.4 \text { (11.0); diagnosed } \\
\text { schizophrenia with antipsychotic } \\
\text { medication; groups: PL (32), PR (33) }\end{array}$ & $\begin{array}{l}\text { Bifiform Balance (2); each } 10^{9} \mathrm{CFU} \text {; } \\
\text { 1/day; tablet }\end{array}$ & Behavioral: PANSS $\leftrightarrow$ \\
\hline Dickerson et al. (2018) [39] & USA & $\begin{array}{l}\text { Acute } \\
\text { Mania }\end{array}$ & 24 & $\begin{array}{l}66(66) ; \text { f: } 63.6 \% \text {; age: } \mathrm{PL} \rightarrow 33.3( \pm 13.3) \\
\text { PR } \rightarrow 37.9 \text { ( } \pm 11.7) \text {; maniac episode; } \\
\text { groups: PL (33), PR (33) }\end{array}$ & Chr. Hansen (2); $>10^{8} \mathrm{CFU} ; 1 /$ day; tablet & Behavioral: YMRS, MADRS, BPRS $\leftrightarrow$ \\
\hline
\end{tabular}


Table 1. Cont.

\begin{tabular}{|c|c|c|c|c|c|c|}
\hline Author (Year) & Country & Subject & $\begin{array}{l}\text { Intake } \\
\text { Length (Week) }\end{array}$ & $\begin{array}{l}\text { SampleSize (A); Sex (f\%); AgeMean } \\
\text { (SD); HealthCondition; Groups (N) }\end{array}$ & $\begin{array}{l}\text { Probiotic Species (N); Dose; Times } \\
\text { Intake per Day; Application }\end{array}$ & Outcomes \\
\hline Diop et al. (2008) [40] & FRA & Stress & 3 & $\begin{array}{l}75 \text { ( } 75) ; f: 72 \% \text {; age: } 38 \pm 11 \text {; healthy; } \\
\text { groups: PL (37), PR ( } 38)\end{array}$ & $\begin{array}{l}\text { Probio stick (2); } 3 \times 10^{9} \mathrm{CFU} \\
\text { 1/day; powder }\end{array}$ & $\begin{array}{l}\text { Behavioral: Questionnaire assessing stress-induced } \\
\text { symptoms ( } 62 \text { items) VAS } 10 \mathrm{~cm}: \downarrow \text { abdominal pain, } \\
\downarrow \text { nausea/vomiting }\end{array}$ \\
\hline Eskandarzadeh et al. (2021) [30] & IRN & $\begin{array}{l}\text { Generalized } \\
\text { Anxiety } \\
\text { Disorder }\end{array}$ & 8 & $\begin{array}{l}48 \text { (39); f: } 81.25 \% \text {; age: } \mathrm{PL} \rightarrow 33.67 \pm 6.56 \\
\mathrm{PR} \rightarrow 34.17 \pm 6.14 \text {; GAD-7 score }>7 ; \\
\text { groups: PL (24), PR (24); Intake with } \\
\text { sertraline in both groups }\end{array}$ & $\begin{array}{l}\text { Takgene Zist Company (4); } 1.8 \times 10^{10} \\
\text { CFU; } 1 \text { /day; capsule }\end{array}$ & $\begin{array}{l}\text { Behavioral: HAM-A } \downarrow \downarrow \text {, State-Anxiety score } \downarrow \text {, } \\
\text { Trait-Anxiety score } \leftrightarrow, \text { BAI } \leftrightarrow, \\
\text { WHO-QOL-BREF } \leftrightarrow\end{array}$ \\
\hline Ghaderi et al. (2019) [41] & IRN & Schizophrenia & 12 & $\begin{array}{l}60 \text { (60); f: } 6.7 \% \text {; age: } \mathrm{PL} \rightarrow 43.2 \pm 6.0, \\
\text { PR } \rightarrow 44.8 \pm 8.3 \text {; diagnosed } \\
\text { schizophrenia; groups: PL (30), PR ( } 30) \text {; } \\
\text { probiotic group within take of } 50,000 \mathrm{IU} \\
\text { of Vitamin D3 every } 2 \text { weeks }\end{array}$ & $\begin{array}{l}\text { Lacto Care (4); each } 2 \times 10^{9} \mathrm{CFU} \\
1 \text {; day; capsule }\end{array}$ & $\begin{array}{l}\text { Behavioral: PANSS } \downarrow \downarrow \text { in general and total subscore } \\
\text { Neuropsychological: BPRS } \leftrightarrow\end{array}$ \\
\hline Gualtieri et al. (2020) [42] & ITA & Anxiety & 12 & $\begin{array}{l}142 \text { (97); f: } 61.9 \% \text {; age: } 41.29( \pm 14.9) \text {; } \\
\text { healthy; groups: PL (71), PR (71) }\end{array}$ & $\begin{array}{l}\text { Biocult Strong, HOMEOSYN (9); each } \\
1.5 \times 10^{10} \mathrm{CFU} / 3 \mathrm{~g} ; 1 / \text { day; powder }\end{array}$ & $\begin{array}{l}\text { Behavioral: HAM-A } \downarrow \downarrow \text { (especially } \downarrow \text { in IL-1 } \beta \\
\text { rs16944 carriers than in non-carrier), BUT } \leftrightarrow \text {, } \\
\text { SCL-90 } \leftrightarrow\end{array}$ \\
\hline Ho et al. (2021) [43] & TWN & Depression & $\begin{array}{l}4 \\
\text { (30 days) }\end{array}$ & $\begin{array}{l}40(40) ; \text { f: } 67.5 \% \text {; age: } \mathrm{PL} \rightarrow 25.47 \pm 4.64, \\
\text { PR } \rightarrow 26.43 \pm 5.95 \text {; PSQI }>5, \text { ISI }>13 \text {; and } \\
\text { met the DSM- } 5 \text { criteria for chronic } \\
\text { primary insomnia; group: PL (19), } \\
\text { PR (21) }\end{array}$ & $\begin{array}{l}\text { Lactobacillus plantarum PS128 (1); } 3 \times \\
10^{10} \mathrm{CFU;} 2 \text { /day; capsule }\end{array}$ & $\begin{array}{l}\text { Behavioral: BDI-II } \downarrow \text {, BAI } \leftrightarrow, \text { PSQI } \leftrightarrow, \text { ISI } \leftrightarrow \text {, ESS } \\
\leftrightarrow, \text { VAS fatigued before sleep } \downarrow \downarrow \\
\text { Neurophysiological: sleep EEG } \leftrightarrow \text { (During N1: } \\
\text { theta power } \% \downarrow \text { at day } 15 \text { ) }\end{array}$ \\
\hline Hwang et al. (2019) [44] & KOR & $\begin{array}{l}\text { Mild } \\
\text { Cognitive } \\
\text { Impairment }\end{array}$ & 12 & $\begin{array}{l}100 \text { (100); f: } 66 \% \text {; age: } \mathrm{PL} \rightarrow 69.2 \text { (7.00), } \\
\text { PR } \rightarrow 68.0 \text { (5.12); healthy with MCI; } \\
\text { groups: PL (50), PR (50) }\end{array}$ & $\begin{array}{l}\text { DW2009 Lactobacillus plantarum C29 (1); } \\
1.25 \times 10^{10} \mathrm{CFU} / \mathrm{g} ; 1 \text { / day; } 2 \text { capsules } \\
(800 \mathrm{mg})\end{array}$ & $\begin{array}{l}\text { Neuropsychological: VLT, ACPT, DST: } \uparrow \text { combined } \\
\text { cognitive function (subscore: Attention/Prefrontal } \\
\text { function } \uparrow \text { in composite score) }\end{array}$ \\
\hline Inoue et al. (2018) [45] & JAP & $\begin{array}{l}\text { Cognitive } \\
\text { Function }\end{array}$ & 12 & $\begin{array}{l}39(38) ; \text { f: } 64.1 \% \text {; age: } 70.3( \pm 3.1) \text {; } \\
\text { healthy; groups: PL (19), PR }(20) \text {; intake } \\
\text { with physical training }\end{array}$ & $\begin{array}{l}\text { Morinaga Milk Industry Co., Ltd. (4); } \\
\text { each } 1.25 \times 10^{10} \mathrm{CFU} ; 1 \text { /day; powder }\end{array}$ & $\begin{array}{l}\text { Behavioral: PHQ-9 } \leftrightarrow, \text { GAD-7 } \leftrightarrow \\
\text { Neuropsychological: MoCA-J } \leftrightarrow\end{array}$ \\
\hline Karbownik et al. (2020) [46] & POL & Stress & 4 & $\begin{array}{l}92 \text { (92); f: } 60 \% \text {; age: } 22.6 \pm 1.3 \text {; healthy; } \\
\text { groups: PL (29), PR-Lactobacillus (32), } \\
\text { PR-Saccharomyces (31) }\end{array}$ & $\begin{array}{l}\text { LacidoEnter }(\mathbf{1}): 5 \times 10^{9} \text {; Dicoflor } 60(\mathbf{1}) \text { : } \\
6 \times 10^{9} ; 1 / \text { day; capsule }\end{array}$ & $\begin{array}{l}\text { Behavioral: STAI } \leftrightarrow \\
\text { Neuropsychological: Performance in Academic } \\
\text { Examination } \leftrightarrow\end{array}$ \\
\hline Kato-Kataoka et al. (2016) [47] & JAP & Stress & 8 & $\begin{array}{l}49 \text { (47); f: } 44.9 \% \text {; age: } \mathrm{PL} \rightarrow 22.8( \pm 0.3) \\
\text { PR } \rightarrow 22.8( \pm 0.4) \text {; healthy; groups: PL } \\
(25), \text { PR }(24)\end{array}$ & $\begin{array}{l}\text { L.casei strain Shirota YIT } 9029(\mathbf{1}) ; 1.0 \times \\
10^{11} \mathrm{CFU} ; 1 \text { /day; liquid }\end{array}$ & $\begin{array}{l}\text { Behavioral: feelings of stress by } 100 \mathrm{~mm} \text { VAS } \downarrow, \\
\text { STAI } \leftrightarrow, \mathrm{NEO}-\mathrm{FFI} \leftrightarrow\end{array}$ \\
\hline
\end{tabular}


Table 1. Cont.

\begin{tabular}{|c|c|c|c|c|c|c|}
\hline Author (Year) & Country & Subject & $\begin{array}{l}\text { Intake } \\
\text { Length (Week) }\end{array}$ & $\begin{array}{l}\text { SampleSize (A); Sex (f\%); AgeMean (SD); } \\
\text { HealthCondition; Groups (N) }\end{array}$ & $\begin{array}{l}\text { Probiotic Species (N); Dose; Times } \\
\text { Intake per Day; Application }\end{array}$ & Outcomes \\
\hline Kazemi et al. (2019) [48] & IRN & Depression & 8 & $\begin{array}{l}110 \text { (110); f: } 70.9 \% \text {; age: } 36.47 \text { (8.03); mild to } \\
\text { moderate major depression; groups: PL (36), } \\
\text { PR (38), PRE (36) }\end{array}$ & $\begin{array}{l}\text { Lallemand Health Solution (2); } \\
>10 \times 10^{9} \text { CFU; } 1 \text { /day; powder }\end{array}$ & Behavioral: BDI $\downarrow$ \\
\hline Kelly et al. (2017) [49] & IRL & $\begin{array}{l}\text { Stress and } \\
\text { Cognitive } \\
\text { Perfor- } \\
\text { mance }\end{array}$ & 8 & $\begin{array}{l}29 \text { (NR); f: } 0 \% \text {; age: } 24.59(0.75) ; \text { healthy; } \\
\text { groups: PL-PR (15), PR-PL (14); RCT } \\
\text { Crossover: switch of treatment after } 4 \text { weeks, } \\
\text { no washout }\end{array}$ & $\begin{array}{l}\text { Lactobacillus rhamnosus (JB-1) (1); } \\
1 \times 10^{9} \mathrm{CFU} ; 1 / \text { day; capsule }\end{array}$ & $\begin{array}{l}\text { Behavioral: BDI, BAI, PSS, STAI, SCL-90, PSQI, } \\
\text { CCL: } \leftrightarrow \\
\text { Neuropsychological: Test from CANTAB battery } \\
\leftrightarrow ; \text { SECPT } \leftrightarrow \\
\text { Neurophysiological: EEG } \leftrightarrow\end{array}$ \\
\hline Kobayashi et al. (2019) [50] & JAP & Memory & 12 & $\begin{array}{l}121 \text { (117); f: } 50.4 \% \text {; age: } P L \rightarrow 61.6 \text { (6.37), } \\
\text { PR } \rightarrow 61.5 \text { (6.83); subjective memory complaints } \\
\text { and MMSE score 22-27; groups: PL (60), } \\
\text { PR (61) }\end{array}$ & $\begin{array}{l}\text { Bifidobacterium breve A1 (1); } \\
>1 \times 10^{10} \mathrm{CFU} ; 1 / \text { day; capsule }\end{array}$ & Neuropsychological: RBANS $\leftrightarrow$, MMSE $\leftrightarrow$ \\
\hline Lew et al. (2019) [51] & MYS & $\begin{array}{l}\text { Stress, } \\
\text { Anxiety, } \\
\text { Memory, } \\
\text { Cognition }\end{array}$ & 12 & $\begin{array}{l}132(103) ; \text { f: } 76.7 \% \text {; age: } \mathrm{PL} \rightarrow 32.1 \pm 11.4 \\
\text { PR } \rightarrow 31.3 \pm 10.8 \text {; healthy with score of } \\
\text { moderate stress level on PSS-10; groups: PL } \\
(51), \text { PR (52) }\end{array}$ & $\begin{array}{l}\text { Lactobacillus plantarum P8 (1); } \\
2 \times 10^{10} \mathrm{CFU;} 1 \text { /day; powder }\end{array}$ & $\begin{array}{l}\text { Behavioral: PSS-10: } \leftrightarrow \text {; DASS- } 42 \text { total: } \downarrow \text { week } 4+ \\
\text { 12; DASS-42 stress: } \downarrow \text { week } 4,8,12 \text {; DASS-42 } \\
\text { anxiety: } \downarrow \text { week } 4,12 ; \text { DASS- } 42 \text { depression } \leftrightarrow \\
\text { Neuropsychological: CBB speed for social } \\
\text { emotional cognition (in women) } \downarrow \downarrow ; \text { international } \\
\text { shopping list memory test } \uparrow\end{array}$ \\
\hline Mohammadi et al. (2016) [53] & IRN & $\begin{array}{l}\text { mental } \\
\text { health }\end{array}$ & 6 & $\begin{array}{l}75 \text { (70); f: } 48.6 \% \text {; age: } \mathrm{PL} \rightarrow 33.1 \pm 6.1 \\
\text { PRyogurt } \rightarrow 33.2 \pm 6.4, \text { PRcapsule } \rightarrow 31.5 \pm 5.8 \\
\text { healthy; groups: PL (20), PRyogurt (25), } \\
\text { PRcapsule (25) }\end{array}$ & $\begin{array}{l}\text { yogurt: Pegah Company (2), } \\
\text { capsules: ZistTakhmir Co. (7); CFU: } \\
\text { different, see Table S1; 1/day }\end{array}$ & Behavioral: GHQ $\leftrightarrow$, DASS $\leftrightarrow$ \\
\hline Murata et al. (2018) [54] & JAP & $\begin{array}{l}\text { Mood } \\
\text { States }\end{array}$ & 12 & $\begin{array}{l}241 \text { (202); f: } 100 \% \text {; age: PL } \rightarrow 20.2 \pm 0.1, \text { PR } \\
(10 \mathrm{LP}) \rightarrow 20.9 \pm 0.5, \text { PR }(30 \mathrm{LP}) \rightarrow 21.1 \pm 0.6 ; \\
\text { healthy; groups: PL (70), PR10LP (69), } \\
\text { PR30LP (63) }\end{array}$ & $\begin{array}{l}\text { LAC-Shield (1); 10PL: } 1 \times 10^{10} \mathrm{CFU} \text {, } \\
\text { 30PL: } 3 \times 10^{10} \mathrm{CFU} ; 1 / \text { day; powder }\end{array}$ & $\begin{array}{l}\text { Behavioral: POMS2 10LP group: } \downarrow \text { T-scores for } \\
\text { Friendliness week } 6+12, \downarrow \text { T-scores for } \\
\text { Vigour-Activity week } 6\end{array}$ \\
\hline Nishida et al. (2017) [55] & JAP & $\begin{array}{l}\text { Stress, } \\
\text { Sleep } \\
\text { Quality }\end{array}$ & 5 & $\begin{array}{l}32 \text { (32); f: } 34.4 \% \text {; age: } \mathrm{PL} \rightarrow 21.31 \pm 0.9 \\
\mathrm{PR} \rightarrow 34.4 \% \text {; healthy; groups: PL (16), PR (16) }\end{array}$ & $\begin{array}{l}\text { heat-inactivated Lactobacillus gasseri } \\
\text { CP2305 (1); } 1 \times 10^{10} \mathrm{CFU} \text {; } \\
\text { 1/day; liquid }\end{array}$ & $\begin{array}{l}\text { Behavioural: GHQ-28 total score } \leftrightarrow, \uparrow \text { somatic } \\
\text { symptom score, significant interaction of treatment } \\
\text { and sex; Zung-SDS } \leftrightarrow ; \text { HADS } \leftrightarrow ; \text { STAI } \leftrightarrow ; \text { PSQI } \leftrightarrow\end{array}$ \\
\hline
\end{tabular}


Table 1. Cont.

\begin{tabular}{|c|c|c|c|c|c|c|}
\hline Author (Year) & Country & Subject & $\begin{array}{l}\text { Intake } \\
\text { Length (Week) }\end{array}$ & $\begin{array}{l}\text { SampleSize (A); Sex (f\%); AgeMean } \\
\text { (SD); HealthCondition; Groups (N) }\end{array}$ & $\begin{array}{l}\text { Probiotic Species (N); Dose; Times } \\
\text { Intake per Day; Application }\end{array}$ & Outcomes \\
\hline Nishida et al. (2019) [56] & JAP & Stress & 24 & $\begin{array}{l}60(\mathrm{NR}) ; \mathrm{f}: 31.7 \% \text {; age: } \mathrm{PL} \rightarrow 25.3 \pm 0.6 \\
\text { PR } \rightarrow 24.9 \pm 0.5 \text {; healthy; groups: PL (31), } \\
\text { PR (29) }\end{array}$ & $\begin{array}{l}\text { Lactobacillus gasseri CP2305 (1); } 1 \times 10^{10} \\
\text { bacterial cells; } 2 \text { tablets } 1 / \text { day }\end{array}$ & $\begin{array}{l}\text { Behavioral: STAI-state } \leftrightarrow, \text { STAI-trait } \downarrow ; \text { GHQ28 } \\
\text { total } \leftrightarrow \text {, depression } \downarrow ; \text { HADS } \leftrightarrow ; \text { PSQI } \downarrow \\
\text { Neurophysiological: EEG: REM and non-REM } \\
\text { sleep times } \leftrightarrow \text {, total delta power } \leftrightarrow, \uparrow \text { ration of } \\
\text { EEG delta power in the first sleep cycle, } \downarrow \text { sleep } \\
\text { latency of the first N3 stage and wake time after } \\
\text { sleep onset }\end{array}$ \\
\hline Nishihira et al. (2014) [57] & JAP & Stress & 12 & $\begin{array}{l}238 \text { (224); f: } 69.2 \% \text {; age: } \mathrm{PL} \rightarrow 54.25 \pm \\
\text { 10.93, PR } \rightarrow 53.61 \pm 11.31 \text {; healthy; } \\
\text { groups: PL (109), PR (115) }\end{array}$ & $\begin{array}{l}\text { MegMilk Snow Brand Co. Ltd. (2); } \\
\text { SBT2055 > } 5 \times 10^{8} \text { CFU, SBT2928 > } \\
1 \times 10^{9} \text { CFU; } 1 / \text { day; yogurt }\end{array}$ & Behavioral: GHQ-28 $\leftrightarrow$ \\
\hline Ohsawa et al. (2018) [58] & JAP & $\begin{array}{l}\text { Cognitive } \\
\text { Function }\end{array}$ & 8 & $\begin{array}{l}61(60) ; \text { f: } 56.7 \% \text {; age: } \mathrm{PL} \rightarrow 57.8 \pm 5.9 \\
\text { PR } \rightarrow 58.5 \pm 6.5 \text {; healthy, baseline } \\
\text { RBANS total score 29-52; groups: PL } \\
\text { (29), PR (31) }\end{array}$ & $\begin{array}{l}\text { Lactobacillus helveticus CM4 (1); dose: NR; } \\
\text { 1/day; liquid }\end{array}$ & $\begin{array}{l}\text { Behavioral: POMS } \leftrightarrow \\
\text { Neuropsychological: RBANS total } \leftrightarrow \text {, five index } \\
\text { scores: } \uparrow \text { attention score, subtest index score: } \\
\uparrow \text { Coding }\end{array}$ \\
\hline $\begin{array}{l}\text { Östlund-Lagerström } \\
\text { et al. (2016) [59] }\end{array}$ & SWE & Wellbeing & 12 & $\begin{array}{l}290 \text { (249); f: PR } \rightarrow 57 \% \text {, PL } \rightarrow 65.6 \% \text {; age: } \\
\text { PR } \rightarrow 72.6 \text { (5.8), PL } \rightarrow 72 \text { (5.6); mentally } \\
\text { and physically fit; groups: PL (124), } \\
\text { PR (125) }\end{array}$ & $\begin{array}{l}\text { Lactobacillus reuteri DSM } 17938 \text { (1); } \\
1 \times 10^{8} \mathrm{CFU} ; 2 \text { /day; powder }\end{array}$ & Behavioral: HADS $\leftrightarrow$, PSS $\leftrightarrow$ \\
\hline Patterson et al. (2020) [60] & DEU & $\begin{array}{l}\text { Stress, } \\
\text { Anxiety }\end{array}$ & 5 & $\begin{array}{l}120 \text { (117); f: } 49.6 \% \text {; age: } \mathrm{PL} \rightarrow 23.25 \text { (4.20), } \\
\text { PR } \rightarrow 23.73 \text { (4.27); healthy; groups: PL } \\
(59), \text { PR (58) }\end{array}$ & $\begin{array}{l}\text { Lacticaseibacillus paracasei Lpc-37 (1); } \\
1.75 \times 10^{10} \mathrm{CFU} ; 1 / \text { day; capsule }\end{array}$ & $\begin{array}{l}\text { Behavioral: STAI } \leftrightarrow \text {, VAS Stress } \leftrightarrow \text {, VAS Insecurity } \\
\leftrightarrow(\downarrow \text { male }), \text { VAS Anxiety } \leftrightarrow(\downarrow \text { male }), \text { VAS } \\
\text { Exhaustion } \leftrightarrow, \text { PSS } \leftrightarrow(\uparrow \text { female), BAI } \leftrightarrow \text {, DASS-24 } \\
\leftrightarrow, \text { Online Diary } \leftrightarrow \text { (Perceived Productivity }+ \\
\text { Perceived Health Status } \uparrow \text { and Sleep Related } \\
\text { Recovery } \uparrow \uparrow \text { in high chronic stress subgroup) }\end{array}$ \\
\hline Rao et al. (2009) [61] & CAN & $\begin{array}{l}\text { Chronic } \\
\text { Fatigue } \\
\text { Syndrome }\end{array}$ & 8 & $\begin{array}{l}39 \text { (35); f: } 77.1 \% \text {; age: } 18-65 \text {; healthy, } \\
\text { diagnostic criteria for CFS; groups: PL } \\
\text { (16), PR (19) }\end{array}$ & $\begin{array}{l}\text { Lactobacillus casei strain Shirota (1); } \\
8 \times 10^{9} \mathrm{CFU} ; 3 / \text { day; powder }\end{array}$ & Behavioral: BDI $\leftrightarrow$, BAI $\downarrow$ \\
\hline
\end{tabular}

60 (60); f: 50\%; age: $\mathrm{PL} \rightarrow 67.3 \pm 11.0$,

$60(60) ; f: 50 \%$; age: $\mathrm{PL} \rightarrow 67.3 \pm 11.0$,
$\mathrm{PR} \rightarrow 71.5 \pm 10.9$; diagnosed with type 2

Raygan et al. (2018) [62] IRN Mental health $12 \quad$ diabetic and coronary heart disease (2-3 LactoCare (4); $8 \times 10^{9} \mathrm{CFU}$; $1 /$ day; application: NR

Behavioral: BDI $\downarrow \downarrow$, BAI $\downarrow \downarrow$, GHQ-28 $\downarrow \downarrow$ 
Table 1. Cont.

\begin{tabular}{|c|c|c|c|c|c|c|}
\hline Author (Year) & Country & Subject & $\begin{array}{l}\text { Intake } \\
\text { Length (Week) }\end{array}$ & $\begin{array}{l}\text { SampleSize (A); Sex (f\%); AgeMean } \\
\text { (SD); HealthCondition; Groups (N) }\end{array}$ & $\begin{array}{l}\text { Probiotic Species (N); Dose; Times } \\
\text { Intake per Day; Application }\end{array}$ & Outcomes \\
\hline Raygan et al. (2019) [63] & IRN & Mental health & 12 & $\begin{array}{l}60(54) ; \text { f: } 61.1 \% \text {; age: } \mathrm{PL} \rightarrow 62.4 \pm 13.1, \\
\text { PR } \rightarrow 64.8 \pm 8.3 \text {; diagnosed with type } 2 \\
\text { diabetic and coronary heart disease }(2-3 \\
\text { vessel); groups: PL (27), PR (27); } \\
\text { Intervention Group with } \\
200 \mu \mathrm{g} / \text { day selenium }\end{array}$ & $\begin{array}{l}\text { LactoCare (4); } 8 \times 10^{9} \mathrm{CFU} ; 1 / \text { day; } \\
\text { application: NR }\end{array}$ & Behavioral: BDI $\downarrow \downarrow$, BAI $\downarrow$, PSQI $\leftrightarrow$ \\
\hline Reininghaus et al.(2020) [64] & AUT & Depression & $\begin{array}{l}4 \\
\text { (28 days) }\end{array}$ & $\begin{array}{l}82 \text { (61); f: } 77.0 \% \text {; age: PL } \rightarrow 40.11 \text { (11.45), } \\
\text { PR } \rightarrow 43.00 \text { (14.31); depressive episode by } \\
\text { MINI; groups: PL (33), PR (28); } \\
\text { bothgroups: } 125 \mathrm{mg} \text { (D-Biotin) Vitamin D7 }\end{array}$ & $\begin{array}{l}\text { OMNi-BiOTiC Stress Repair }(9) \\
7.5 \times 10^{9} \mathrm{CFU} ; 1 \text { /day; powder }\end{array}$ & $\begin{array}{l}\text { Behavioral: HAMD, BDI-II, SCL-90R, MSS, } \\
\text { GLQI: } \leftrightarrow\end{array}$ \\
\hline Roman et al. (2018) [65] & ESP & Fibromyalgia & 8 & $\begin{array}{l}40 \text { (31); f: } 92.5 \% \text {; age: } \mathrm{PL} \rightarrow 50.27 \pm 7.86 \\
\text { PR } \rightarrow 55.00 \pm 8.37 \text {; diagnosed with FMS; } \\
\text { groups: PL (20), PR (20) }\end{array}$ & $\begin{array}{l}\text { ERGYPHILUS Plus (4); } 6 \times 10^{6} \mathrm{CFU} \text {; } \\
\text { 4/day; capsule }\end{array}$ & $\begin{array}{l}\text { Behavioral: STAI } \leftrightarrow, \text { BDI } \leftrightarrow \\
\text { Neuropsychological: MMSE } \leftrightarrow \text {, two-choice } \\
\text { task } \downarrow \text {, IGT } \leftrightarrow\end{array}$ \\
\hline Romijn et al. (2017) [66] & NZL & Depression & 8 & $\begin{array}{l}79 \text { (79); f: } 78.5 \% \text {; age: } \mathrm{PL} \rightarrow 35.1 \text { (14.5), } \\
\text { PR } \rightarrow 35.8 \text { (14); healthy; } \geq 11 \text { on QIDS-SR16 } \\
\text { or } \geq 14 \text { on DASS-42; groups: PL (39), } \\
\text { PR }(40)\end{array}$ & $\begin{array}{l}\text { Lallemand Health Solution (2); } \\
\geq 3 \times 10^{9} \mathrm{CFU} ; 1 / \text { day; powder }\end{array}$ & $\begin{array}{l}\text { Behavioral: MADRS, iCGI, QIDS-SR16, GAF, } \\
\text { DASS-42: } \leftrightarrow\end{array}$ \\
\hline Rudzki et al. (2019) [67] & POL & Depression & 8 & $\begin{array}{l}79(60) ; \text { f: } 71.7 \% \text {; age: } \mathrm{PL} \rightarrow 38.9(12), \\
\text { PR } \rightarrow 39.13 \text { (9.96); major depression during } \\
\text { SSRI monotherapy or drug free; groups: } \\
\text { PL (39), PR (40); with SSRI treatment }\end{array}$ & $\begin{array}{l}\text { Sanprobi IBS (1); } 10 \times 10^{9} \mathrm{CFU} \text {; } \\
\text { 2/day; capsule }\end{array}$ & $\begin{array}{l}\text { Behavioral: HAM-D } 17 \leftrightarrow, \text { SCL-90 } \leftrightarrow, \\
\text { PSS-10 } \leftrightarrow \\
\text { Neuropsychological: APT } \uparrow \uparrow, \text { Stroop Test A + } \\
\text { B } \leftrightarrow, \text { RFFT } \leftrightarrow, \text { TMT A }+ \text { B } \leftrightarrow, \text { CVLT } \uparrow\end{array}$ \\
\hline Sanchez et al. (2017) [68] & CAN & $\begin{array}{l}\text { Depression, } \\
\text { Anxiety and } \\
\text { Stress }\end{array}$ & 24 & $\begin{array}{l}126(125) ; \mathrm{f}: 61.6 \% \text {; age: } \mathrm{PL} \rightarrow 37 \pm 10 \\
\mathrm{PR} \rightarrow 35 \pm 10 ; \mathrm{BMI} \text { between } 29 \text { and } 41 \\
\mathrm{~kg} / \mathrm{m} \text {; groups: PL (63), PR (62) }\end{array}$ & $\begin{array}{l}\text { Lactobacillus rhamnosus } \\
\text { CGMCC1.3724 (1); } 3.24 \times 10^{8} \\
\text { CFU/day; } 2 \text { /day; capsules }\end{array}$ & Behavioral: BDI $\downarrow$, STAI $\leftrightarrow$, PSS $\leftrightarrow$ \\
\hline Sashihara et al. (2013) [69] & JAP & $\begin{array}{l}\text { Mental } \\
\text { Condition }\end{array}$ & 4 & $\begin{array}{l}44 \text { ( } 44) ; \mathrm{f}: 0 \% \text {; age: } \mathrm{PL} \rightarrow 20.2 \pm 1.1, \\
\mathrm{PR}+\alpha \mathrm{LA} \rightarrow 19.9 \pm 0.9, \mathrm{PR} \rightarrow 19.8 \pm 0.9 ; \\
\text { engaged in high-intensity training } \geq 5 \\
\text { days/week; groups: PL (14), } \mathrm{PR}(15), \\
\text { PR }+\alpha \mathrm{LA} \text { (15) }\end{array}$ & $\begin{array}{l}\text { Lactobacillus gasseri OLL2809 (1); } \\
1 \times 10^{10} \mathrm{CFU} ; 2 \text { tablets } 3 / \text { day }\end{array}$ & Behavioral: POMS and VAS for fatigue $\leftrightarrow$ \\
\hline
\end{tabular}


Table 1. Cont.

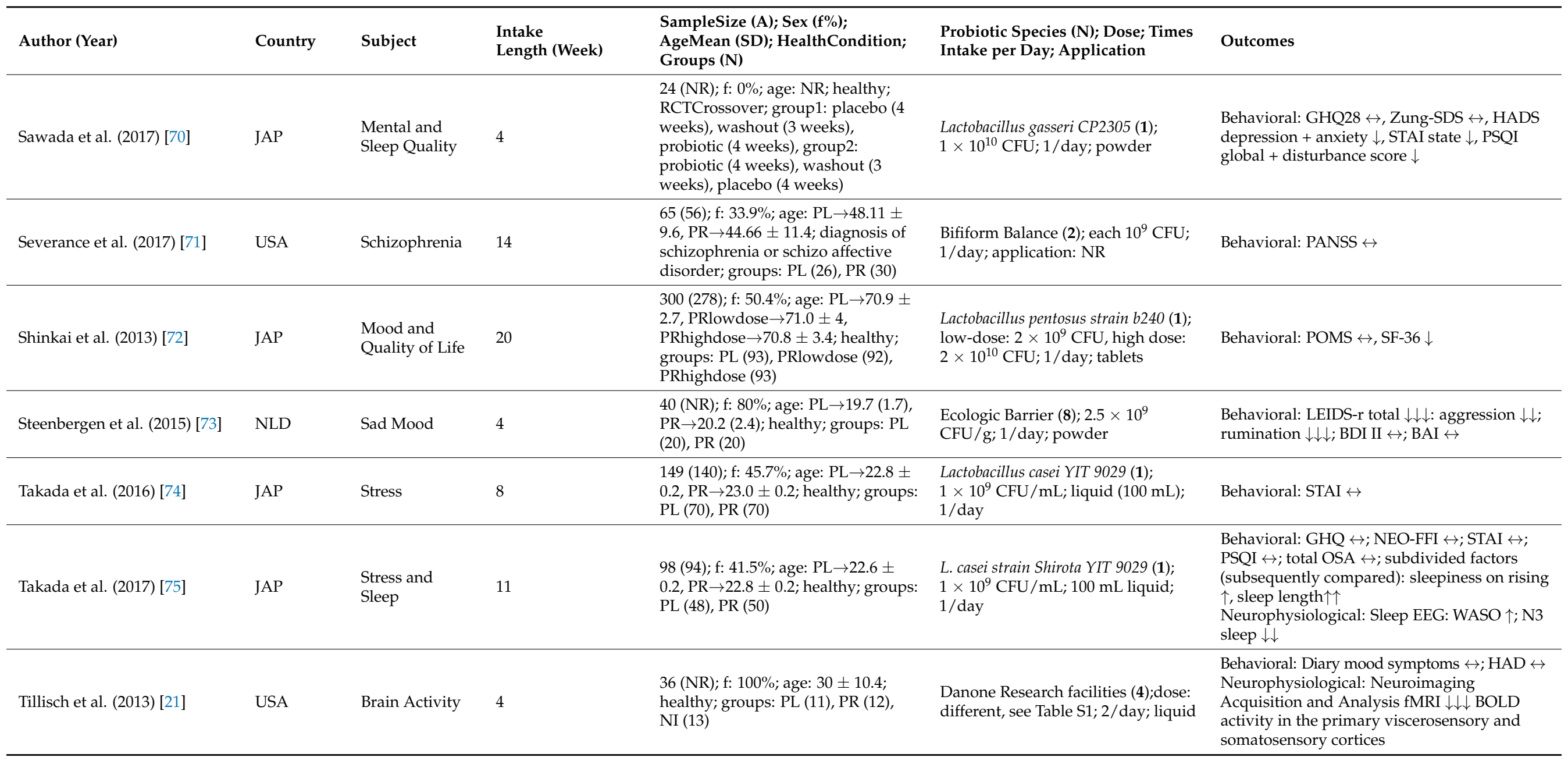


Table 1. Cont.

\begin{tabular}{|c|c|c|c|c|c|c|}
\hline Author (Year) & Country & Subject & $\begin{array}{l}\text { Intake } \\
\text { Length (Week) }\end{array}$ & $\begin{array}{l}\text { SampleSize (A); Sex (f\%); AgeMean } \\
\text { (SD); HealthCondition; Groups (N) }\end{array}$ & $\begin{array}{l}\text { Probiotic Species (N); Dose; Times } \\
\text { Intake per Day; Application }\end{array}$ & Outcomes \\
\hline Tran et al. (2019) [76] & USA & Anxiety & $\begin{array}{l}4 \\
\text { (28 days) }\end{array}$ & $\begin{array}{l}90 \text { (68); f: } 75.6 \% \text {; age: } 20.59(2.65) ; \\
\text { healthy; ConditionA (17): } \\
\text { highCFU[50billion] + high species count } \\
\text { [18], ConditionB (19): } \\
\text { highCFU[50billion] + low species count } \\
\text { [10], ConditionC (16): control/placebo } \\
\text { group, ConditionD (19): } \\
\text { lowCFU[15billion] + high species } \\
\text { count[18], ConditionE (19): } \\
\text { lowCFU[10billion] + low species } \\
\text { count [10] }\end{array}$ & $\begin{array}{l}\text { commercially available as } \\
\text { over-the-counter products (e.g., } \\
\text { Amazon) (from } 10 \text { till 20); } 1 \times 10^{10}-5 \\
\times 10^{10} \text { CFU; } 1 \text { /day; pills }\end{array}$ & $\begin{array}{l}\text { Behavioural: BAI, ACQ-R, PANAS, NMR, } \\
\text { PSWQ } \leftrightarrow\end{array}$ \\
\hline Wang et al. (2019) [22] & DEU & Stress & 4 & $\begin{array}{l}43(40) ; f: 65 \% \text {; age: } P L \rightarrow 33.00 \pm 2.83 \\
\text { PR } \rightarrow 31.00 \pm 2.28 \text {; healthy; groups: PL } \\
(20), P R(20)\end{array}$ & $\begin{array}{l}\text { Bifidobacterium longum } 1714 \text { (1); } \\
1 \times 109 \text { CFU; } 1 \text { /day; powder }\end{array}$ & $\begin{array}{l}\text { Behavioural: SF- } 36 \leftrightarrow \\
\text { Neurophysiological: resting state MEG: } \uparrow \text { theta } \\
\text { band power, } \downarrow \text { beta- } 3 \text { band power in different } \\
\text { brain region; during social distress: } \uparrow(S) \text { theta } \\
\text { band power, } \uparrow \text { alpha band power in different } \\
\text { brain region; in both conditions' } \\
\text { inclusion/exclusion; NTS } \leftrightarrow ; \text { SEP } \leftrightarrow ; \text { MQ } \leftrightarrow\end{array}$ \\
\hline Xiao et al. (2020) [77] & JAP & Memory & 16 & $\begin{array}{l}80 \text { (80); f: } 51.3 \% \text {; age: PL } \rightarrow 60.9 \text { (6.9), } \\
\text { PR } \rightarrow 61.3 \text { (7.7); MMSE score } 22 \text { o rmore; } \\
\text { group: PL (40), PR (40) }\end{array}$ & $\begin{array}{l}\text { Bifidobacterium breve A1 (1); } \\
1 \times 10^{10} \mathrm{CFU} ; 1 / \text { day; capsule }\end{array}$ & $\begin{array}{l}\text { Neuropsychological: RBANS } \downarrow \downarrow \downarrow \text { in RBANS } \\
\text { total score, Immediate memory, } \\
\text { Visuospatial/Constructional, Delayed } \\
\text { memory; JMCIS } \leftrightarrow\end{array}$ \\
\hline Yamamura et al. (2009) [78] & JAP & Sleep & 3 & $\begin{array}{l}30 \text { (29); f: } 20.7 \% \text {; age: PL } \rightarrow 70.6 \pm 5.65, \\
\text { PR } \rightarrow 72.14 \pm 5.67 \text {; healthy, no use of } \\
\text { substances that influence sleep; groups: } \\
\text { PLfirst (15), PRfirst (14); } \\
\text { RCTCrossover } \rightarrow \text { placebo first group: } \\
\text { placebo ( } 3 \text { weeks), washout ( } 3 \text { weeks), } \\
\text { probiotic ( } 3 \text { weeks), probiotic first group: } \\
\text { probiotic ( } 3 \text { weeks), washout (3 weeks), } \\
\text { placebo ( } 3 \text { weeks) }\end{array}$ & $\begin{array}{l}\text { Lactobacillus helveticus strain CM4 (1); } \\
\text { dose: NR; } 1 \text { /day; liquid }\end{array}$ & $\begin{array}{l}\text { Behavioural: SHRI } \leftrightarrow, \text { SF- } 36 \leftrightarrow \\
\text { Neurophysiological: Actigraphy } \leftrightarrow\end{array}$ \\
\hline Zhang et al. (2021) [79] & $\mathrm{CHN}$ & Depression & 9 & $\begin{array}{l}82(69) \text {; f: } 63.8 \% \text {; age: } \mathrm{PL} \rightarrow 49.7 \pm 9.6 \\
\mathrm{PR} \rightarrow 45.8 \pm 12.3 \text {; diagnosed depression } \\
\text { (DSM-5); groups: PL (31), PR (38) }\end{array}$ & $\begin{array}{l}\text { Lacticaseibacillus paracasei YIT } 9029 \\
\text { (strain Shirota: LcS) (1); } \\
1.0 \times 10^{10} \text { CFU; } 1 \text { /day; liquid }\end{array}$ & $\begin{array}{l}\text { Behavioural: BDI, HAMD: decreased } \\
\text { significantly in both groups, no comparison } \\
\text { between groups }\end{array}$ \\
\hline
\end{tabular}


Tak Gen Zist Pharmaceutical Company (Tehran, Iran) provided or sponsored six trials from the sample $(11.1 \%)$, as well as Yakult (Minato, Japan) $(n=6 ; 11.5 \%)$, Lallemand Institute (Montréal, QC, Canada) $(n=6 ; 11.1 \%)$, Winclove Probiotics (Amsterdam, The Netherlands) ( $n=5 ; 9.3 \%)$, Asahi Group Holdings (Sumida, Japan) $(n=4 ; 7.7 \%)$, Alimentary Health (Cork, Ireland) $(n=3 ; 5.6 \%)$, Morinaga Milk Industry (Minato-ku, Japan) $(n=3 ; 5.6 \%)$, Pfizer (New York, NY, USA) $(n=2 ; 3.7 \%)$ and HOMEOSYN (Rome, Italy) $(n=2 ; 3.7 \%)$. From 54 studies in this sample, 37 studies were sponsored or provided by these few manufacturers (68.5\%). Among the 15 studies from Japan, $10(66.7 \%)$ were provided from Yakult $(n=3 ; 20 \%)$, Asahi Group Holdings $(n=4 ; 26.7 \%)$ and Morinaga Milk Industry $(n=3 ; 20 \%)$. In Iran, six out of seven studies $(85.7 \%)$ were provided by Tak Gen Zist Pharmaceutical Company.

For the meta-analysis, 3017 participants out of 30 trials were eligible. There were 2563 participants at the end of the trials and the data of 2595 participants were analyzed. The median age was $36.2(22.8-51.4)$ years and $62.9 \%$ were women. The intervention periods ranged between 4 and 24 weeks. The median length was 8 (4.3-12) weeks. Most probiotics were ingested as powder $(n=8 ; 26.7 \%)$ and were either single-strains $(n=16 ; 53.3 \%)$ or multi-strains $(n=14 ; 46.7 \%)$. The latter consisted of two to twenty different probiotic strains. The intake ranged from one to four servings per day. Mostly, the intake was once per day $(n=24 ; 80 \%)$. The median number of colony-forming units (CFU) was $9 \times 10^{9}\left(3.5 \times 10^{9}-2 \times 10^{10}\right)$ CFU per day with a range from $2.4 \times 10^{7}$ to $1.4 \times 10^{11} \mathrm{CFU}$ per day. An overview of characteristics is provided Table S3.

\subsection{Summary of Study Outcomes}

Overall, the heterogeneity of studies was high. Many different questionnaires were used to describe different outcomes with regard to behavioral or neuropsychological aspects. Table S4 shows the most common questionnaires in the review sample and Table S5 specifically for the meta-analytical sample with their results of the comparison between probiotic and placebo group for every single study. An overview across studies at the qualitative level is presented in Figure 2. One study did not report their results differentiated by groups [79] and among the others which reported their results, most studies found no significant differences between the probiotic treatment and the placebo groups, often only stating this finding and not reporting the figures.

Figure 3 shows the results at qualitative level for the questionnaires eligible for metaanalysis where the data were available from the publication or provided by the authors upon request.

\subsubsection{Mood States and Stress}

Mood states: The Profile of Mood States (POMS) questionnaire was investigated by four studies and at qualitative level no differences between the probiotic and placebo groups were observed. In the meta-analysis, as shown in Figure 4, the placebo interventions were in all subscales slightly in favor when compared to the probiotic groups, while the heterogeneity was low and never higher than $\mathrm{I}^{2}=13 \%$. All participants were healthy and without any diagnosed psychiatric disorder.

Stress: Similar to mood, none of the five studies found superiority of the probiotic intervention when applying the Perceived Stress scale (PSS) at qualitative level. With the meta-analysis (Figure 5), the probiotic interventions were slightly in favor when compared to the placebo groups (mean PSS difference -0.17 (95\% CI: $-0.33,-0.00) ; p=0.05)$, while the heterogeneity was very low with $\mathrm{I}^{2}=0 \%$. All participants were healthy and without any diagnosed psychiatric disorder. 


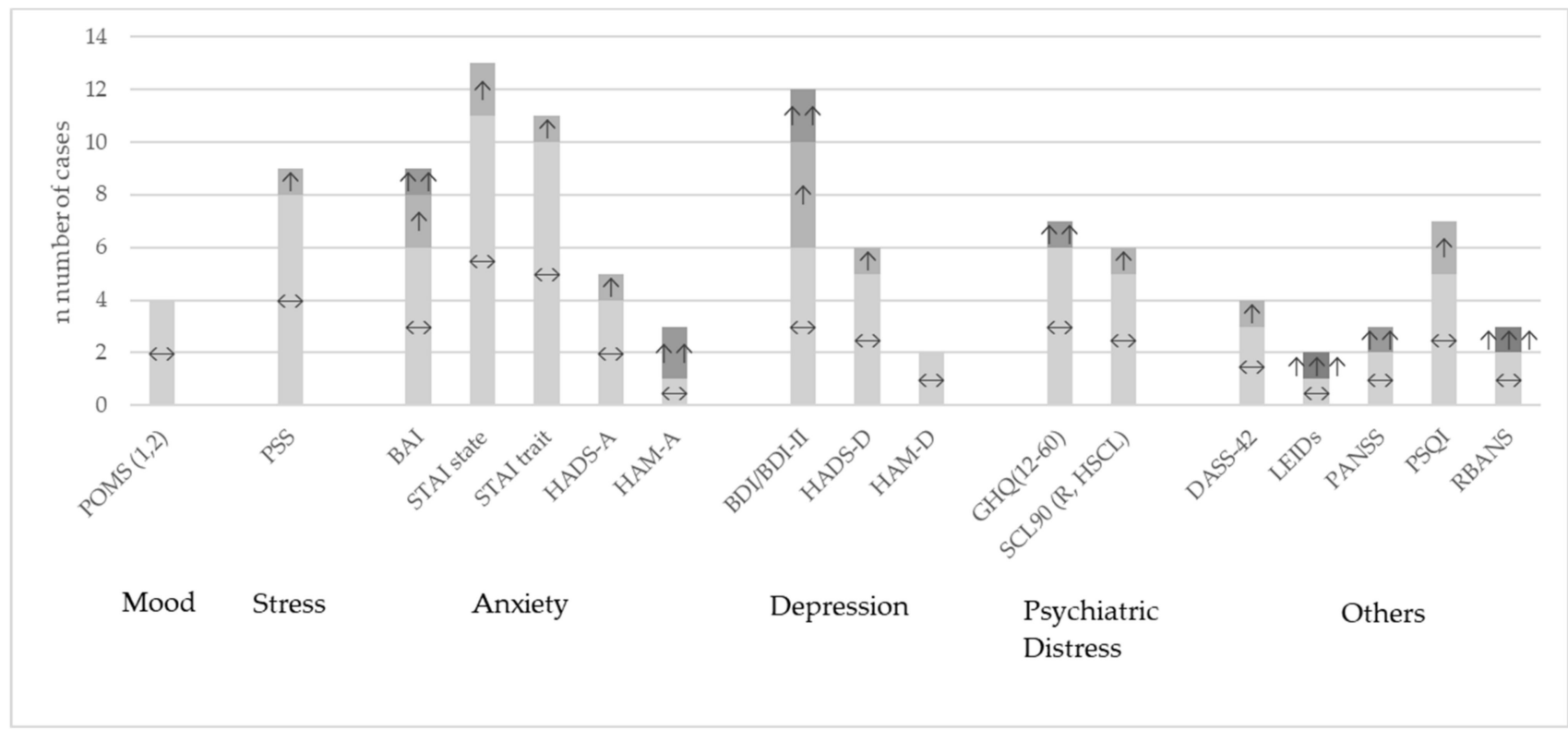

Figure 2. Study outcomes at qualitative level for the most used questionnaires compared between probiotic versus placebo intervention. $\leftrightarrow$ : no significant differences between the groups; $\uparrow:$ improvement $p<0.05$; $\uparrow$ : improvement $p<0.01 ; \uparrow \uparrow \uparrow:$ improvement $p<0.001$; BAI: Beck Anxiety Inventory; BDI: Beck Depression Inventory; DASS-42: Depression, Anxiety and Stress Scale; GHQ: General Health Questionnaire; HADS-A/-D: Hospital Anxiety and Depression Scale; HAM-A: Hamilton Anxiety Rating Scale; HAM-D: Hamilton Depression Rating Scale; LEIDs: Leiden index of depression severity; POMS: Profile of Mood States; PSQI: Pittsburgh Sleep Quality Index; PSS: Perceived Stress Scale; RBANS: Repeatable Battery for the Assessment of Neuropsychological Status; SCL90: Symptoms Checklist-90; STAI: State Trait Anxiety Inventory.

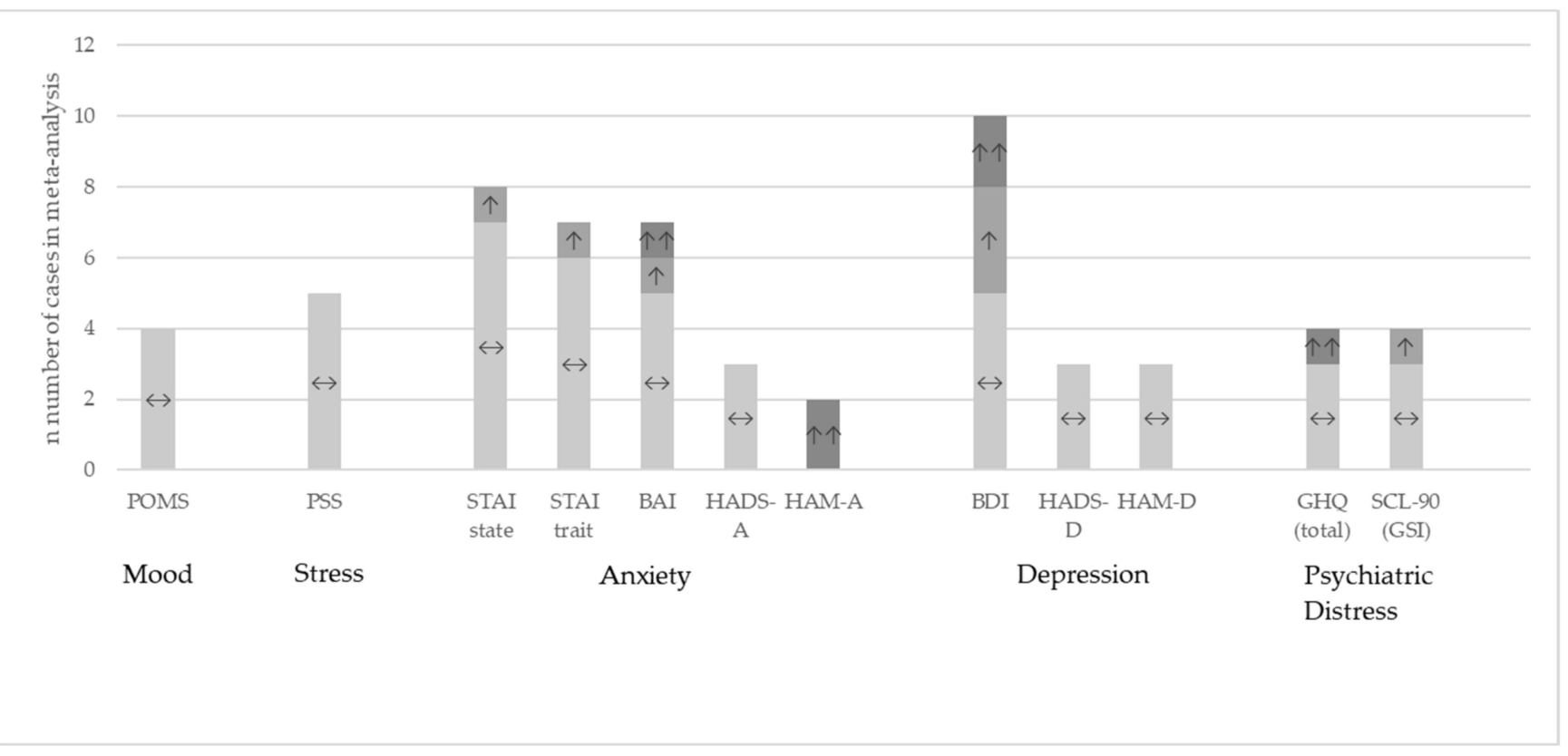

Figure 3. Study outcomes at qualitative level for the studies included in the meta-analysis. Comparison between the probiotic and placebo group $[20,30,32,33,37,38,41,42,44-49,51,52,55,57,58,63,64,66-72,77,78]$. $\leftrightarrow$ : no significant differences between the groups; $\uparrow:$ significant improvement in the probiotic group versus placebo $p<0.05 ; \uparrow \uparrow$ : significant improvement in the probiotic group versus placebo $p<0.01$. 


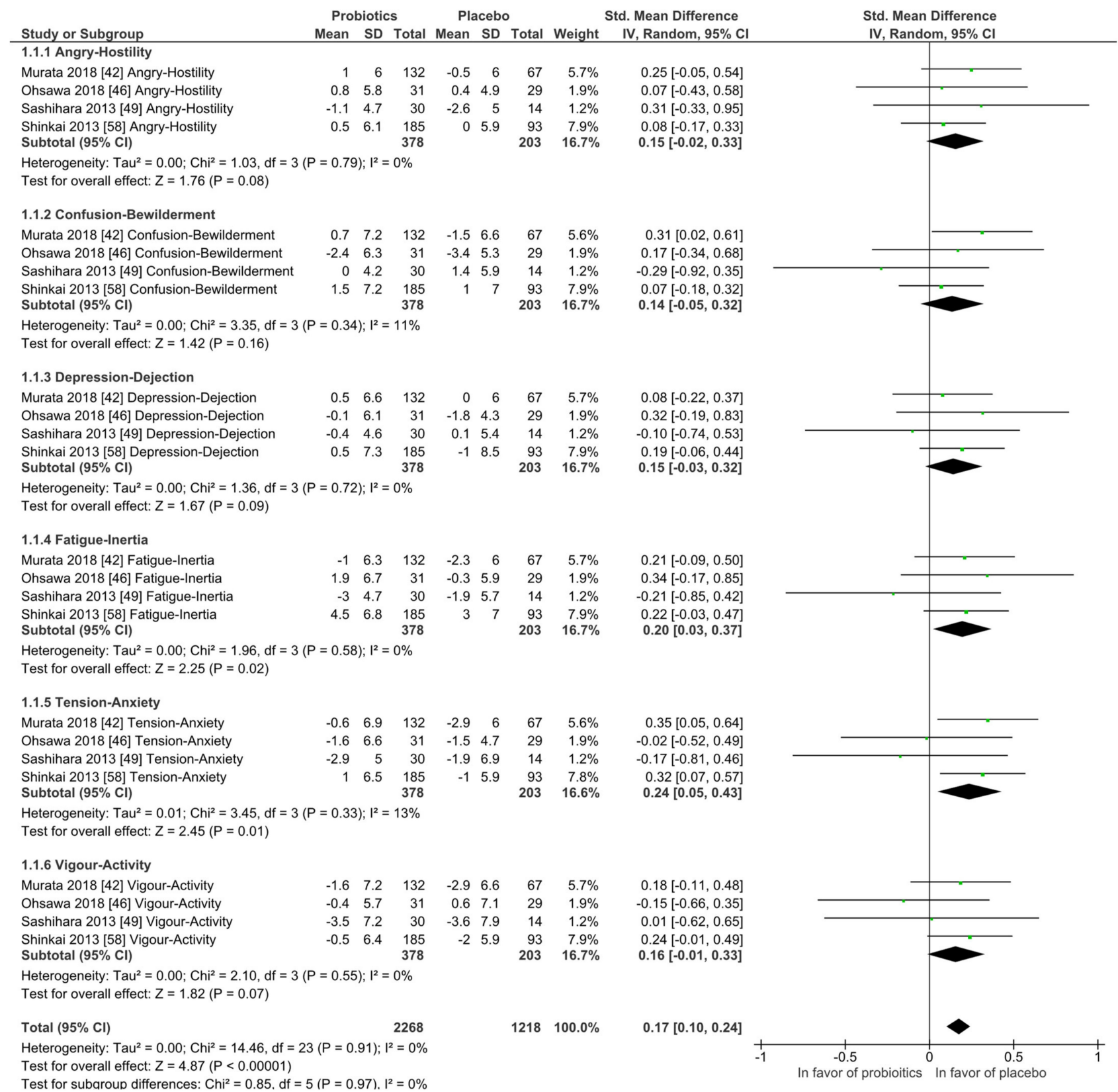

Figure 4. Quantitative analysis for Profile of Mood States (POMS) of randomized controlled trials receiving either probiotics or placebo treatment. Murata 2018 [54], Ohsawa 2018 [58], Sashihara 2013 [69], Shinkai 2013 [72].

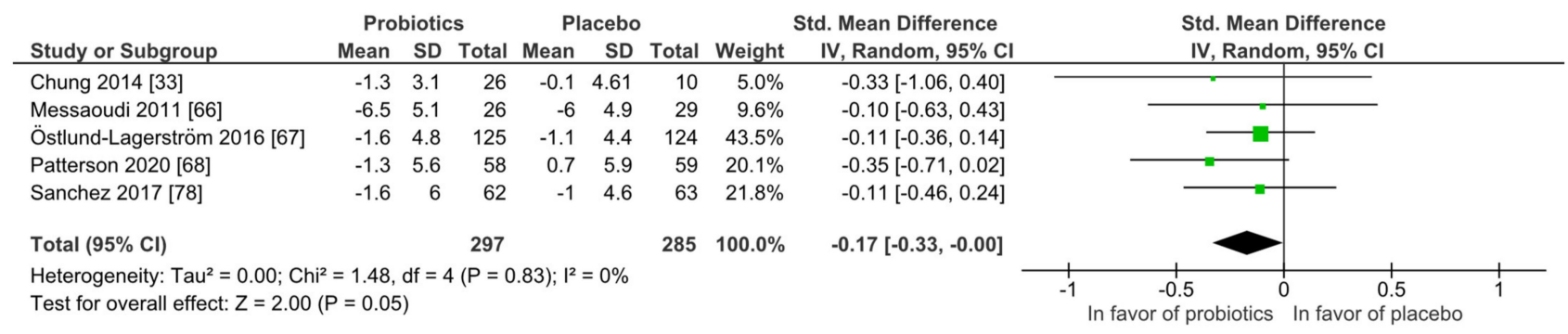

Figure 5. Quantitative analysis for Perceived Stress scale (PSS) of randomized controlled trials receiving either probiotics or placebo treatment. Chung 2014 [36], Messaoudi 2011 [52], ÖstlundLagerström 2016 [59], Patterson 2020 [60], Sanchez 2017 [68]. 


\subsubsection{Anxiety, Depression and Psychiatric Distress}

Anxiety: Overall, anxiety improved in both groups, regardless of group allocation. The most used questionnaires for detecting anxiety in the study samples when data were available were the Beck Anxiety Inventory (BAI), the Hospital Anxiety Scale (HADS-A), the Hamilton Anxiety Rating Scale (HAM-A) and the State-Trait Anxiety Inventory (STAI; state and trait score). From the review sample of 54 trials, the mentioned questionnaires were collected and the results presented for 41 instances from 23 studies. The reason for this is that six studies used different questionnaires for the same outcome $[30,49,55,56,60,70]$.

For the group comparisons (probiotic versus placebo treatment) at qualitative level, 32 instances $(78 \%)$ showed no differences between the groups and nine studies were in favor of the probiotic group (22\%). Of these, one study was conducted in patients with diagnosed anxiety [30]. Here several questionnaires were applied and no difference between groups was reported for BAI and STAI trait, but there was superiority for the probiotic intervention for the STAI state.

For quantitative analysis, 17 studies remained and the results are presented as forest plots in Figure 6. The probiotic intervention was not in favor compared to the placebo group (mean difference -0.30 (95\% CI: $-0.60,0.01) ; p=0.06$ ) accompanied with high heterogeneity $\left(\mathrm{I}^{2}=86 \%\right)$. To reduce heterogeneity, subgroup analyses were performed for study length, probiotic strain numbers, country, application form and health status as presented in Figures S1-S6, not changing the outcome of the full analysis presented in Figure 6. Heterogeneity dropped considerably only in the single-strain subgroup with $\mathrm{I}^{2}=52 \%$.

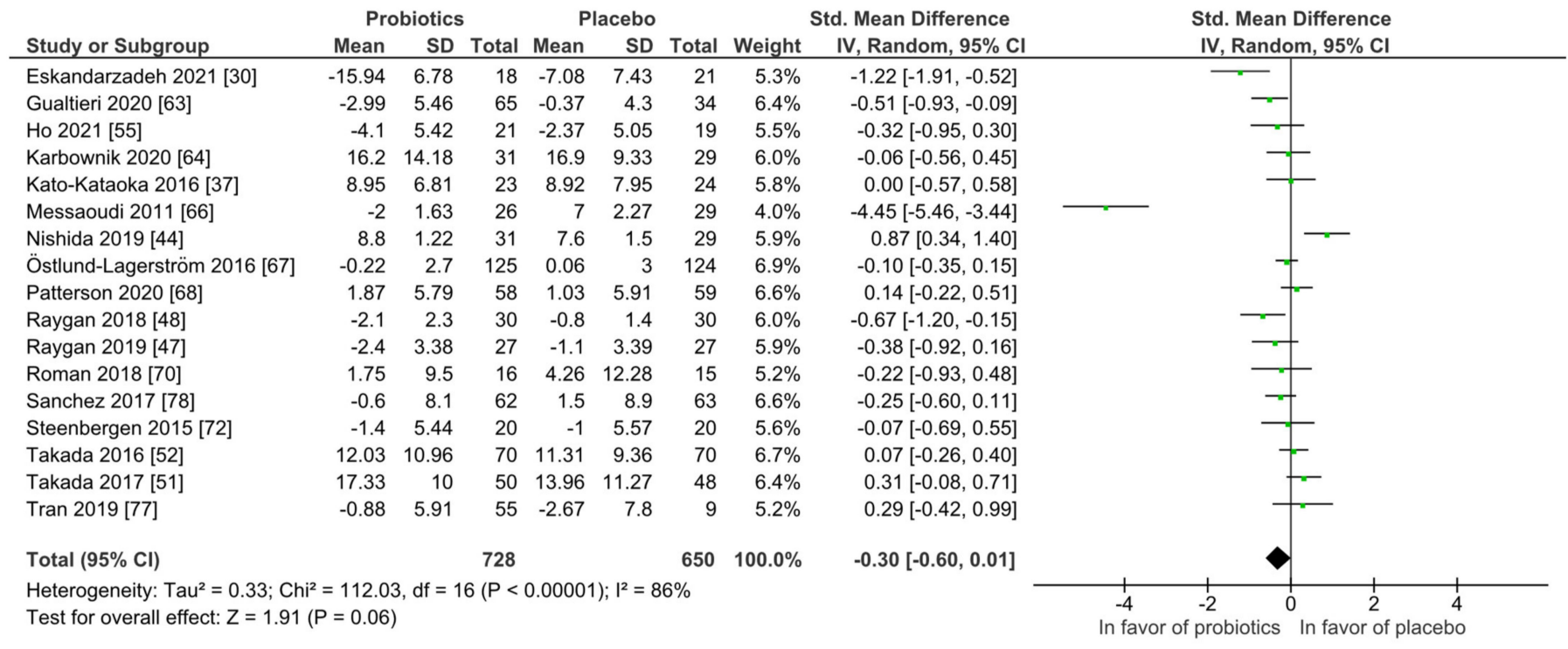

Figure 6. Quantitative analysis for anxiety of randomized controlled trials receiving either probiotics or placebo treatment. Eskandarzadeh 2021 [30], Gualtieri 2020 [42], Ho 2021 [43], Karbownik 2020 [46], Kato-Kataoka 2016 [47], Messaoudi 2011 [52], Nishida 2019 [56], Östlund-Lagerström 2016 [59], Patterson 2020 [60], Raygan 2018 [62], Raygan 2019 [63], Roman 2018 [65], Sanchez 2017 [68], Steenbergen 2015 [73], Takada 2016 [74], Takada 2017 [75], Tran 2019 [76].

Depression: Overall, depression improved in both groups, regardless of group allocation. The most used questionnaires for detecting depression were the Beck Depression Inventory (BDI), Hospital Depression Scale (HADS-D) and Hamilton Depression Rating Scale (HAM-D). From the review sample of 54 trials, 19 studies reported findings for the above-mentioned questionnaires. Among these, one study used more than one questionnaire for the same outcome [64].

For the group comparisons at qualitative level, 12 studies (63\%) showed no differences between the groups and 7 studies were in favor of the probiotic group $(37 \%)$. Of these, 
4 studies were conducted in patients with diagnosed depression. Half of the studies found no group differences and the other half favored the probiotic group.

For quantitative analysis, 15 studies were available and the results are presented as forest plots in Figure 7. The probiotic interventions were favorable when compared to the placebo groups (mean difference -0.37 (95\% CI: $-0.55,-0.20) ; p \leq 0.0001$ ) with small effect size, while the heterogeneity was moderate with $\mathrm{I}^{2}=48 \%$. For a differentiated understanding of the findings, subgroup analyses for probiotic strain numbers, country, application form and health status were performed and presented in Figures S7-S12. Heterogeneity decreased remarkably in the multi-strain subgroup $\left(\mathrm{I}^{2}=4 \%\right)$, the Asia subgroup $\left(\mathrm{I}^{2}=0 \%\right)$ and the subgroup with diagnosed depression disorder $\left(\mathrm{I}^{2}=0 \%\right)$.

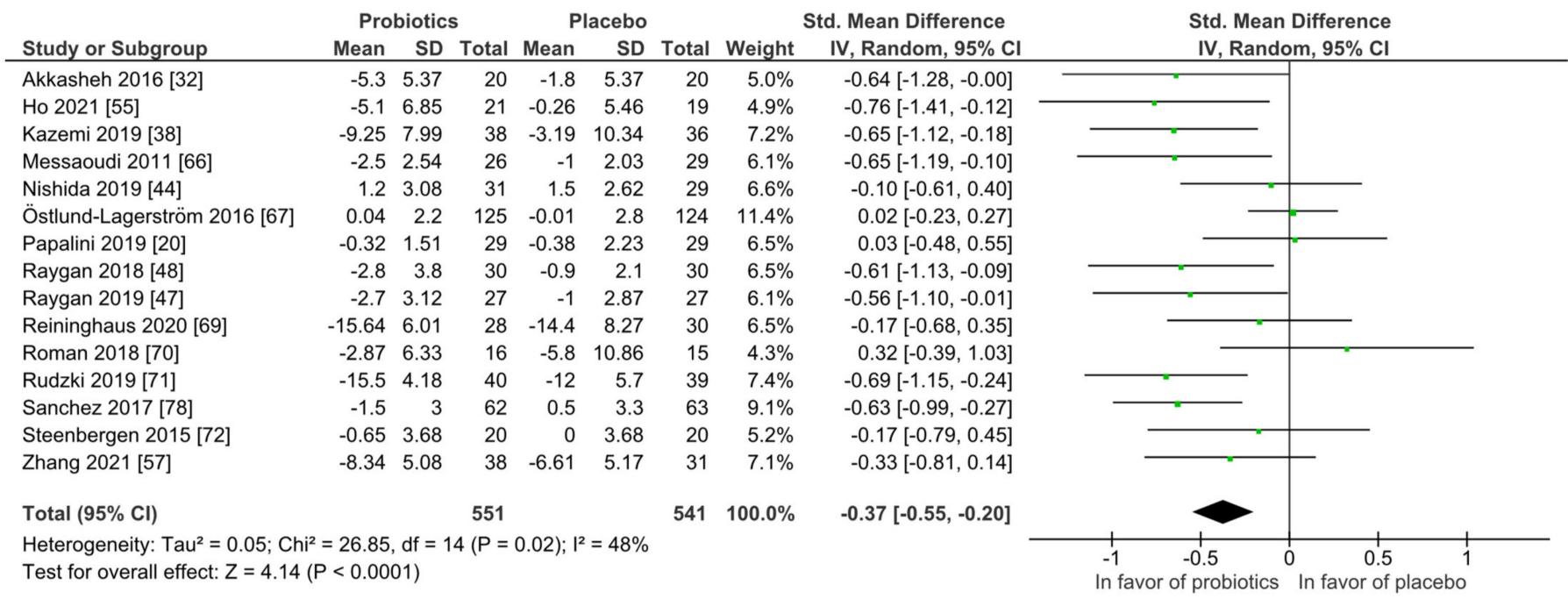

Figure 7. Quantitative analysis for depression of randomized controlled trials receiving either probiotics or placebo treatment. Akkasheh 2016 [32], Ho 2021 [43], Kazemi 2019 [48], Messaoudi 2011 [52], Nishida 2019 [56], Östlund-Lagerström 2016 [59], Papalini 2019 [20], Raygan 2018 [62], Raygan 2019 [63], Reininghaus 2020 [64], Roman 2018 [65], Rudzki 2019 [67], Sanchez 2017 [68], Steenbergen 2015 [73], Zhang 2021 [79].

Psychiatric Distress: Overall, psychiatric distress improved in both groups, regardless of group allocation. The most used questionnaires for detecting psychiatric distress were the General Health Questionnaire (GHQ) and the Symptoms Checklist90 (SCL-90). From the review sample of 54 trials, 14 studies reported findings of the above-mentioned questionnaires.

For the group comparisons (probiotic versus placebo treatment) at qualitative level, 12 studies $(85.7 \%)$ showed no differences between the groups and two studies were in favor of the probiotic group (14.3\%). Of these, one study was conducted in patients with diagnosed depression and no group differences were reported.

For quantitative analysis, eight studies were available, and the results are presented as forest plots in Figure 8. The probiotic interventions were favorable when compared to the placebo groups (mean difference -0.33 (95\% CI: $-0.53,-0.13) ; p=0.001$ ) with small effect size, while the heterogeneity was moderate with $\mathrm{I}^{2}=36 \%$. For a differentiated understanding of the findings, subgroup analyses for study strain numbers, country, application form and health status were performed and presented in Figures S13-S18. Heterogeneity decreased remarkably in the short-term treatment subgroup of 4 to 8 weeks $\left(\mathrm{I}^{2}=0 \%\right)$, and the subgroup with the application form capsules / pills $\left(\mathrm{I}^{2}=0 \%\right)$. 


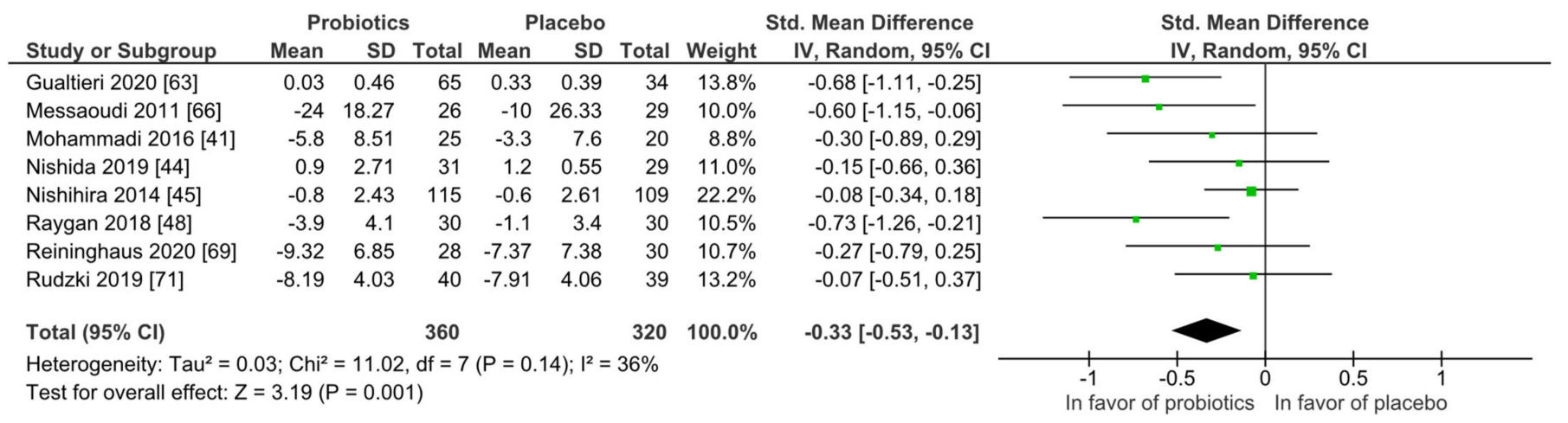

Figure 8. Overview of psychiatric distress symptom outcomes according to questionnaire. Gualtieri 2020 [42], Messaoudi 2011 [52], Mohammadi 2016 [53], Nishida 2019 [56], Nishihira 2014 [57], Raygan 2018 [62], Reininghaus2020 [64], Rudzki 2019 [67].

\subsubsection{Central Nervous System (CNS) Findings in EEG and Brain Imaging}

Six studies recorded EEG, three of them during sleep to assess the effects of probiotics on sleep parameters $[43,56,75]$; these studies reported improvements of sleep quality. Two further studies found that probiotic intake improved memory function [34] and modulated brain regions associated with relaxation and attention [33]. In contrast, Kelly et al. (2017) found no significant difference between the probiotic and placebo treatment in the EEG measurements for cognitive functions in hypothalamic-pituitary adrenal response while socially evaluated cold pressor test, paired associates learning, attention witching task, emotional troop and emotion recognition task [49].

In total, five imaging studies were found. Tillisch et al. (2013) investigated the effects of a multi-strain probiotic using fMRI during identification of emotional face expressions and found that probiotic treatment affected brain activity of regions that control central processing of emotion and sensation [21]. Bagga et al. $(2018,2019)$ published two fMRI studies using the same multi-strain probiotic and found that the probiotic improved memory performance and altered brain activation patterns [19] and that behavior was modulated towards a shift of efficient attentional control and changes in functional, but not structural, connectivity [18]. Papalini et al. (2019) investigated the effects of a multi-strain probiotic by fMRI using the emotional face-matching, emotional face-word Stroop and the classic color-word Stroop paradigms and found no difference between probiotic and placebo treatment. [20]. Finally, the next generation probiotic Bifidobacterium longum 1714 was tested under social stress (octracism) conditions by (Wang et al. (2019)) by applying the cyber ball game and was proved to reduce stress effects in a magnetoencephalography paradigm [22].

\subsection{Risk of Bias}

The risk of bias assessment is presented in Table 2. The overall risk of bias was low in 18 studies (33.3\%), with some concerns in 18 studies (33.3\%) and high in 18 studies (33.3\%). Overall, the studies with intention-to-treat-analysis (ITT), there was a low risk of bias in 11 studies (42.3\%), some concerns in 7 studies (26.9\%) and in 8 studies high (30.8\%). While in the trials with per-protocol-analysis (PP), 7 studies had a low bias (25\%), 11 had some concerns $(39.3 \%)$ and in 10 studies the risk of bias was high $(35.7 \%)$. 
Table 2. Risk of Bias. +: Low risk, !: Some concerns, -: High risk, D1: Randomisation process, D2: Deviations from the intended interventions, D3: Missing outcome data, D4: Measurement of the outcome, D5: Selection of the reported result.

\begin{tabular}{|c|c|c|c|c|c|c|}
\hline Intention-to-Treat & $\underline{\mathrm{D} 1}$ & $\underline{\mathrm{D} 2}$ & $\underline{\mathrm{D} 3}$ & $\underline{\mathrm{D} 4}$ & $\underline{\text { D5 }}$ & Overall \\
\hline Akkasheh 2016 [32] & + & & & + & + & \\
\hline Allen 2016 [59] & & & & & P & \\
\hline Bagga 2018 [19] & + & & & & ! & \\
\hline Bagga 2019 [18] & + & & & & $!$ & \\
\hline Benton 2007 [60] & + & & & & ! & \\
\hline De Lorenzo 2017 [61] & + & & & & + & \\
\hline Dickerson 2018 [73] & + & & ! & & + & \\
\hline Diop 2008 [62] & + & & & & ! & \\
\hline Ghaderi 2019 [34] & $!$ & & & & + & \\
\hline Ho 2021 [55] & + & & & & + & \\
\hline Hwang 2019 [35] & + & & & & + & \\
\hline Karbownik 2020 [64] & + & & & & + & \\
\hline Kato-Kataoka 2016 [37] & + & & & & + & \\
\hline Kazemi 2019 [38] & + & & & & + & \\
\hline Kelly 2017 [65] & + & & & 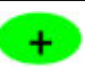 & $!$ & \\
\hline Nishida 2017 [43] & + & & & $!$ & $!$ & \\
\hline Nishida 2019 [44] & + & & & & + & \\
\hline Östlund-Lagerström 2016 [67] & $!$ & & & 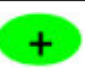 & + & ! \\
\hline Patterson $2020[68]$ & + & 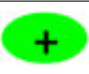 & 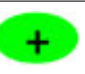 & 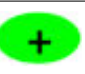 & + & \\
\hline Raygan 2018 [48] & + & & & & + & \\
\hline Romijn 2017 [79] & $!$ & P & 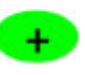 & P & + & ! \\
\hline Sanchez 2017 [78] & + & D & + & + & + & \\
\hline Sashihara 2013 [49] & + & P & + & + & $!$ & ! \\
\hline Sawada 2017 [50] & + & & & + & $!$ & \\
\hline Steenbergen 2015 [72] & + & & $!$ & + & $!$ & \\
\hline Xiao 2020 [56] & + & + & + & + & + & \\
\hline
\end{tabular}


Table 2. Cont.

\begin{tabular}{|c|c|c|c|c|c|c|}
\hline Per-protocol & $\underline{\text { D1 }}$ & $\underline{\text { D2 }}$ & $\underline{\mathrm{D} 3}$ & $\underline{\mathrm{D} 4}$ & $\underline{\text { D5 }}$ & Overall \\
\hline Adikari 2020 [54] & + & & & + & + & \\
\hline Chung 2014 [33] & + & & & + & $!$ & \\
\hline Dickerson 2014 [74] & + & & & & + & \\
\hline Eskandarzadeh 2021 [30] & + & & & & + & \\
\hline Gualtieri 2020 [63] & + & & & & + & \\
\hline Inoue 2018 [36] & + & & & & + & \\
\hline Kobayashi 2019 [39] & + & & & & + & \\
\hline Lew 2019 [40] & + & &. & & + & \\
\hline Messaoudi 2011 [66] & + & & & & + & \\
\hline Mohammadi 2016 [41] & + & & & & + & \\
\hline Murata 2018 [42] & + & & & & + & \\
\hline Nishihira 2014 [45] & + & & & & $!$ & ) \\
\hline Ohsawa 2018 [46] & $!$ & & & & + & ) \\
\hline Papalini 2019 [20] & $!$ & & & & + & ! \\
\hline Rao 2009 [75] & + & & & & $!$ & 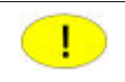 \\
\hline Raygan 2019 [47] & + & & & & + & \\
\hline Reininghaus2020 [69] & $!$ & & & 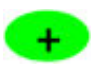 & + & \\
\hline Roman 2018 [70] & $!$ & & & & + & \\
\hline Romijn 2017 [79] & + & & $!$ & & + & \\
\hline Rudzki 2019 [71] & + & & $!$ & & + & \\
\hline Shinkai 2013 [58] & + & ! & + & + & + & ! \\
\hline Takada 2016 [52] & + & + & + & + & $!$ & ! \\
\hline Takada 2017 [51] & + & + & + & + & + & ! \\
\hline Tillisch 2013 [21] & + & & & + & $!$ & \\
\hline Tran 2019 [77] & + & + & $!$ & + & $!$ & ! \\
\hline Wang 2019 [22] & + & $!$ & + & + & + & ! \\
\hline
\end{tabular}


Table 2. Cont.

\begin{tabular}{|c|c|c|c|c|}
\hline Per-protocol & D2 & D4 & D5 & Overall \\
\hline \multicolumn{5}{|c|}{ Yamamura 2009 [53] } \\
\hline \multicolumn{5}{|l|}{ Zhang 2021 [57] } \\
\hline & Low risk & & & \\
\hline ! & Some concerns & & & \\
\hline & High risk & & & \\
\hline D1 & Randomisation process & & & \\
\hline D2 & Deviations from the intended interventions & & & \\
\hline D3 & Missing outcome data & & & \\
\hline D4 & Measurement of the outcome & & & \\
\hline D5 & Selection of the reported result & & & \\
\hline
\end{tabular}

For studies included in the meta-analysis, the overall risk of bias was low in 9 studies $(30 \%)$, with some concern in 9 studies $(30 \%)$ and high in 12 studies $(40 \%)$. Eighteen of the trials were analyzed per protocol and not per intention-to-treat. While most of the intentionto-treat studies remained with low risk of bias ( 5 of $12,41.7 \%$ ), the per-protocol studies had six studies with some concerns (33.3\%) and eight with a high risk of bias $(44.4 \%)$.

\section{Discussion}

This systematic review and meta-analysis investigated the effects of probiotic treatment on (i) psychological well-being (mood and stress), (ii) psychiatric symptoms (anxiety, depression, psychiatric distress) and (iii) central functions.

At qualitative level we found that probiotic treatment mostly was not superior to placebo treatment when evaluating at questionnaire level. Only a fraction of these studies was included in meta-analysis following quality of study assessment.

Regarding mood and stress, at qualitative level the studies showed no superiority of the probiotic intervention and at quantitative level, placebo was slightly in favor for mood and the probiotics for stress, suggesting that psychological well-being at this level was not affected. Similarly, for psychiatric symptoms including anxiety, depression and psychiatric distress, most studies found no group differences at qualitative level. In line with this, at the quantitative level, no differences between groups were found for anxiety and, regarding depression and psychiatric distress, the probiotic group was only slightly in favor. However, also bearing in mind the findings at the qualitative level, the clinical relevance of these findings is questionable. Nevertheless, it needs to be taken in account that only a fraction of studies included a patient collective. For patients with diagnosed depression, the small effect of probiotic treatment on symptom improvement was stable when considering the substantially low heterogeneity.

In addition, EEG and imaging studies summarized in this review proved that probiotics are able to also exert effects on CNS function in humans and not only in pre-clinical models, although the number of studies is still low. Thus, there appears to be potential for probiotics in this area for prevention and treatment, but to which extent and in which specific areas such an approach is promising is unclear to date.

Experience from extensive probiotic research in irritable bowel syndrome and other GIrelated disciplines over the last four decades shows that the results are still conflicting due to several reasons. One is that most probiotics are marketed as nutritional supplements [80] and not as drugs [12]. As a consequence of this, clinical trials usually do not match all standards imposed by the US Food and Drug Administration (FDA) or European Medical Agency (EMA) [80]. Furthermore, not all probiotics can be expected to be of similar efficacy (number of strains, type of strain(s)) [81], and they may not be effective across 
different patient collectives and even patient subgroups [12]. What is more, in the field of probiotic research in the GI-related disciplines, effects of probiotics on psyche were not thoroughly investigated in the past and, if reported, no effects were observed [26]. At this point it should be considered that if probiotics exert clinical meaningful psychological effects, it is unlikely that no one has noticed this within 40 years of research. However, it needs to be also considered that these probiotics were not clearly defined for targeting CNS function. Additionally, many traditional multi-strain products are used with the interactions between the strains having been rarely or not at all investigated. The multistrain probiotics mostly have their origin in the milk industry where probiotic mixtures were composed due to practical reasons. Probiotic research has the potential to improve in all fields, with next generation probiotics being now available. These probiotics are derived from next generation sequencing and are selected for specific properties and specific indication [82]. In this area of research, next generation probiotics selected and developed for their ability to improve psychiatric condition and potentially other CNS functions are suitable. In this review, with regard to the imaging studies, only one study investigated the effects of such a next generation probiotic and found stress-reducing effects under social stress conditions [22]. In IBS (reviewed elsewhere [26]), one more next generation probiotic was tested using fMRI and found reduced limbic reactivity towards fearful stimuli [23]. Both studies hint at the potential of these probiotics for modulating mood and stress in health and disease. Whether or not they have the potential to also influence pathological mental health states is completely unclear. If yes, it is most likely that probiotics themselves may not deliver satisfying results, warranting a multicomponent treatment.

This study has strength and limitations. A clear strength is the methodological approach taken according to PRISMA criteria. To provide homogeneity of the trials, the search was limited to RCTs in adults only using probiotics and postbiotics, but neither synbiotics nor prebiotics. Still, despite clear eligibility criteria, the studies were heterogeneous regarding study design, probiotic strain(s), application form and duration, sample population and outcomes. Since many studies were not able to report positive outcomes for the probiotic intervention, the data were often not reported and the result was only mentioned in the text, not allowing us to include the findings in the meta-analysis. Thus, we contacted the corresponding authors at least three times to obtain as many data for meta-analysis as possible. However, we were not always successful, and two studies could not provide the data because the sponsor had not agreed. To be as objective as possible, we therefore always reported the data at both the qualitative and quantitative level. Overall, the heterogeneity of the meta-analyses was acceptable and only for anxiety were the findings high. To reduce heterogeneity and for a more detailed understanding of the data, subgroup analyses were performed. These further reduced heterogeneity in several cases and also allowed us to get insights on the role of participant status (patient versus healthy) in these analyses.

\section{Conclusions}

Overall, we found that probiotics have the potential to exert effects on CNS function in humans. However, most probiotics did not affect mood, stress, anxiety, depression and psychiatric distress when compared to placebo at the qualitative level. At quantitative level, depression and psychiatric distress improved slightly in the probiotic condition. It remains unclear to which extent next generation probiotics selected and developed for their ability to improve psychiatric condition and potentially other CNS functions (sometimes called psychobiotics), are beneficial for improving mental well-being and psychiatric symptoms in health and disease.

Supplementary Materials: The following supporting information can be downloaded at: https: / / www.mdpi.com/article/10.3390/nu14030621/s1, Text S1: Search Strategy, Table S1: Bacterial Species of Probiotics, Table S2: Questionnaires and tests applied by the included studies, Table S3: Study characteristics across studies, Table S4: Overview of the most used questionnaires and their results of all included studies, Table S5: Study outcomes at qualitative level for the most used questionnaires of the studies included in the meta-analysis, Figure S1: Effect of intake period on anxiety of randomized 
controlled trials receiving either probiotics or placebo treatment, Figure S2: Effect of single-strain versus multi-strain probiotics on Anxiety in randomized controlled trials receiving either probiotics or placebo treatment, Figure S3: Effect of studies from Asia versus studies from Europe on anxiety in randomized controlled trials receiving either probiotics or placebo treatment, Figure S4: Effect of application form on anxiety of randomized controlled trials receiving either probiotics or placebo treatment, Figure S5: Effect of studies with healthy participants on anxiety in randomized controlled trials receiving either probiotics or placebo treatment, Figure S6: Overview of anxiety symptom outcomes according to questionnaire, Figure S7: Effect of intake period on depression of randomized controlled trials receiving either probiotics or placebo treatment, Figure S8: Effect of single-strain versus multi-strain probiotics on depression in randomized controlled trials receiving either probiotics or placebo treatment, Figure S9: Effect of studies from Asia versus studies from Europe on depression in randomized controlled trials receiving either probiotics or placebo treatment, Figure S10: Effect of application form on depression of randomized controlled trials receiving either probiotics or placebo treatment, Figure S11: Effect of studies with healthy participants on depression in randomized controlled trials receiving either probiotics or placebo treatment, Figure S12: Overview of depression symptom outcomes according to questionnaire, Figure S13: Effect of intake period on psychiatric distress of randomized controlled trials receiving either probiotics or placebo treatment, Figure S14: Effect of single-strain versus multi-strain probiotics on psychiatric distress in randomized controlled trials receiving either probiotics or placebo treatment, Figure S15: Effect of studies from Asia versus studies from Europe on psychiatric distress in randomized controlled trials receiving either probiotics or placebo treatment, Figure S16: Effect of application form on psychiatric distress of randomized controlled trials receiving either probiotics or placebo treatment, Figure S17: Effect of studies with healthy participants on psychiatric distress in randomized controlled trials receiving either probiotics or placebo treatment, Figure S18: Overview of psychiatric distress symptom outcomes according to questionnaire.

Author Contributions: Conceptualization, I.M., C.L.M.d.S. and P.E.; methodology, all authors; software, C.L.M.d.S., C.H.; validation, C.L.M.d.S., C.H., I.M.; formal analysis, C.L.M.d.S.; data curation, C.L.M.d.S., C.H.; writing-original draft preparation, C.L.M.d.S., I.M.; writing — review and editing, all authors; visualization, C.L.M.d.S., C.H.; supervision, I.M. and P.E. All authors have read and agreed to the published version of the manuscript.

Funding: I.M. acknowledges support by the German Aerospace Center (DLR), grant number 50WB1920. All authors thank the Open Access Publishing Fund of Tübingen University for support.

Institutional Review Board Statement: Not applicable.

Informed Consent Statement: Not applicable.

Data Availability Statement: All data are reported in the manuscript and the Supplementary Materials.

Conflicts of Interest: The authors C.L.M.d.S., C.H. and I.M. declare no conflict of interest. P.E. is a consultant of Alimentary Health, Aptiny, Arena, Cemet, Indigo, SymbioPharm, and 4DPharma. He is also in the speaker oards of Alimentary Health, Biocodex, Biogen, Indigo, MDC, Medice, Merz, and Sanof. The funders had no role in the design of the study; in the collection, analyses or interpretation of data; in the writing of the manuscript, or in the decision to publish the results.

\section{References}

1. The Lancet Global, H. Mental health matters. Lancet Glob. Health 2020, 8, e1352. [CrossRef]

2. Clarke, D.M.; Currie, K.C. Depression, anxiety and their relationship with chronic diseases: A review of the epidemiology, risk and treatment evidence. Med. J. Aust. 2009, 190, S54-S60. [CrossRef] [PubMed]

3. Cryan, J.F.; O'Riordan, K.J.; Sandhu, K.; Peterson, V.; Dinan, T.G. The gut microbiome in neurological disorders. Lancet Neurol. 2020, 19, 179-194. [CrossRef]

4. Thursby, E.; Juge, N. Introduction to the human gut microbiota. Biochem. J. 2017, 474, 1823-1836. [CrossRef] [PubMed]

5. Caso, J.R.; MacDowell, K.S.; González-Pinto, A.; García, S.; de Diego-Adeliño, J.; Carceller-Sindreu, M.; Sarramea, F.; CaballeroVillarraso, J.; Gracia-García, P.; De la Cámara, C.; et al. Gut microbiota, innate immune pathways, and inflammatory control mechanisms in patients with major depressive disorder. Transl. Psychiatry 2021, 11, 645. [CrossRef] [PubMed]

6. Rajoka, M.S.R.; Mehwish, H.M.; Kitazawa, H.; Barba, F.J.; Berthelot, L.; Umair, M.; Zhu, Q.; He, Z.; Zhao, L. Techno-functional properties and immunomodulatory potential of exopolysaccharide from Lactiplantibacillus plantarum MM89 isolated from human breast milk. Food Chem. 2021, 377, 131954. [CrossRef] [PubMed] 
7. Carding, S.; Verbeke, K.; Vipond, D.T.; Corfe, B.M.; Owen, L.J. Dysbiosis of the gut microbiota in disease. Microb. Ecol. Health Dis. 2015, 26, 26191. [CrossRef] [PubMed]

8. Sekirov, I.; Russell, S.L.; Antunes, L.C.M.; Finlay, B.B. Gut Microbiota in Health and Disease. Physiol. Rev. 2010, 90, 859-904. [CrossRef] [PubMed]

9. Wang, H.; Lee, I.S.; Braun, C.; Enck, P. Effect of Probiotics on Central Nervous System Functions in Animals and Humans: A Systematic Review. J. Neurogastroenterol. Motil. 2016, 22, 589-605. [CrossRef] [PubMed]

10. Dinan, T.G.; Cryan, J.F. Brain-Gut-Microbiota Axis and Mental Health. Psychosom. Med. 2017, 79, 920-926. [CrossRef] [PubMed]

11. Socała, K.; Doboszewska, U.; Szopa, A.; Serefko, A.; Włodarczyk, M.; Zielińska, A.; Poleszak, E.; Fichna, J.; Wlaź, P. The role of microbiota-gut-brain axis in neuropsychiatric and neurological disorders. Pharmacol. Res. 2021, 172, 105840. [CrossRef]

12. Mack, I.; Schwille-Kiuntke, J.; Mazurak, N.; Niesler, B.; Zimmermann, K.; Mönnikes, H.; Enck, P. A Nonviable Probiotic in Irritable Bowel Syndrome: A Randomized, Double-Blind, Placebo-Controlled, Multicenter Study. Clin. Gastroenterol. Hepatol. 2021. [CrossRef]

13. Akter, S.; Park, J.H.; Jung, H.K. Potential Health-Promoting Benefits of Paraprobiotics, Inactivated Probiotic Cells. J. Microbiol. Biotechnol. 2020, 30, 477-481. [CrossRef]

14. Sakandar, H.A.; Zhang, H. Trends in Probiotic(s)-Fermented milks and their in vivo functionality: A review. Trends Food Sci. Technol. 2021, 110, 55-65. [CrossRef]

15. Reis, D.J.; Ilardi, S.S.; Punt, S.E.W. The anxiolytic effect of probiotics: A systematic review and meta-analysis of the clinical and preclinical literature. PLoS ONE 2018, 13, e0199041. [CrossRef]

16. Chudzik, A.; Orzyłowska, A.; Rola, R.; Stanisz, G.J. Probiotics, Prebiotics and Postbiotics on Mitigation of Depression Symptoms: Modulation of the Brain-Gut-Microbiome Axis. Biomolecules 2021, 11, 1000. [CrossRef]

17. Riaz Rajoka, M.S.; Thirumdas, R.; Mehwish, H.M.; Umair, M.; Khurshid, M.; Hayat, H.F.; Phimolsiripol, Y.; Pallarés, N.; MartíQuijal, F.J.; Barba, F.J. Role of Food Antioxidants in Modulating Gut Microbial Communities: Novel Understandings in Intestinal Oxidative Stress Damage and Their Impact on Host Health. Antioxidants 2021, 10, 1563. [CrossRef]

18. Bagga, D.; Aigner, C.S.; Reichert, J.L.; Cecchetto, C.; Fischmeister, F.P.S.; Holzer, P.; Moissl-Eichinger, C.; Schöpf, V. Influence of 4-week multi-strain probiotic administration on resting-state functional connectivity in healthy volunteers. Eur. J. Nutr. 2019, 58, 1821-1827. [CrossRef]

19. Bagga, D.; Reichert, J.L.; Koschutnig, K.; Aigner, C.S.; Holzer, P.; Koskinen, K.; Moissl-Eichinger, C.; Schöpf, V. Probiotics drive gut microbiome triggering emotional brain signatures. Gut Microbes 2018, 9, 486-496. [CrossRef]

20. Papalini, S.; Michels, F.; Kohn, N.; Wegman, J.; van Hemert, S.; Roelofs, K.; Arias-Vasquez, A.; Aarts, E. Stress matters: Randomized controlled trial on the effect of probiotics on neurocognition. Neurobiol. Stress 2019, 10, 100141. [CrossRef]

21. Tillisch, K.; Labus, J.; Kilpatrick, L.; Jiang, Z.; Stains, J.; Ebrat, B.; Guyonnet, D.; Legrain-Raspaud, S.; Trotin, B.; Naliboff, B.; et al. Consumption of fermented milk product with probiotic modulates brain activity. Gastroenterology 2013, 144, 1394-1401.E4. [CrossRef] [PubMed]

22. Wang, H.; Braun, C.; Murphy, E.F.; Enck, P. Bifidobacterium longum $1714^{\mathrm{TM}}$ Strain Modulates Brain Activity of Healthy Volunteers During Social Stress. Am. J. Gastroenterol. 2019, 114, 1152-1162. [CrossRef] [PubMed]

23. Pinto-Sanchez, M.I.; Hall, G.B.; Ghajar, K.; Nardelli, A.; Bolino, C.; Lau, J.T.; Martin, F.P.; Cominetti, O.; Welsh, C.; Rieder, A.; et al. Probiotic Bifidobacterium longum NCC3001 Reduces Depression Scores and Alters Brain Activity: A Pilot Study in Patients With Irritable Bowel Syndrome. Gastroenterology 2017, 153, 448-459.E8. [CrossRef] [PubMed]

24. Liberati, A.; Altman, D.G.; Tetzlaff, J.; Mulrow, C.; Gøtzsche, P.C.; Ioannidis, J.P.; Clarke, M.; Devereaux, P.J.; Kleijnen, J.; Moher, D. The PRISMA statement for reporting systematic reviews and meta-analyses of studies that evaluate healthcare interventions: Explanation and elaboration. BMJ 2009, 339, b2700. [CrossRef]

25. Schardt, C.; Adams, M.B.; Owens, T.; Keitz, S.; Fontelo, P. Utilization of the PICO framework to improve searching PubMed for clinical questions. BMC Med. Inform. Decis. Mak. 2007, 7, 16. [CrossRef]

26. Le Morvan de Sequeira, C.; Kaeber, M.; Cekin, S.E.; Enck, P.; Mack, I. The Effect of Probiotics on Quality of Life, Depression and Anxiety in Patients with Irritable Bowel Syndrome: A Systematic Review and Meta-Analysis. J. Clin. Med. 2021, $10,3497$. [CrossRef]

27. DerSimonian, R.; Laird, N. Meta-analysis in clinical trials. Control. Clin. Trials 1986, 7, 177-188. [CrossRef]

28. Normand, S.L. Meta-analysis: Formulating, evaluating, combining, and reporting. Stat. Med. 1999, 18, 321-359. [CrossRef]

29. Review Manager (RevMan), Version 5.4; The Cochrane Collaboration: London, UK, 2020.

30. Eskandarzadeh, S.; Effatpanah, M.; Khosravi-Darani, K.; Askari, R.; Hosseini, A.F.; Reisian, M.; Jazayeri, S. Efficacy of a multispecies probiotic as adjunctive therapy in generalized anxiety disorder: A double blind, randomized, placebo-controlled trial. Nutr. Neurosci. 2021, 24, 102-108. [CrossRef]

31. Sterne, J.A.C.; Savović, J.; Page, M.J.; Elbers, R.G.; Blencowe, N.S.; Boutron, I.; Cates, C.J.; Cheng, H.Y.; Corbett, M.S.; Eldridge, S.M.; et al. RoB 2: A revised tool for assessing risk of bias in randomised trials. BMJ 2019, 366, 14898. [CrossRef]

32. Akkasheh, G.; Kashani-Poor, Z.; Tajabadi-Ebrahimi, M.; Jafari, P.; Akbari, H.; Taghizadeh, M.; Memarzadeh, M.R.; Asemi, Z.; Esmaillzadeh, A. Clinical and metabolic response to probiotic administration in patients with major depressive disorder: A randomized, double-blind, placebo-controlled trial. Nutrition 2016, 32, 315-320. [CrossRef]

33. Adikari, A.; Appukutty, M.; Kuan, G. Effects of Daily Probiotics Supplementation on Anxiety Induced Physiological Parameters among Competitive Football Players. Nutrients 2020, 12, 1920. [CrossRef] 
34. Allen, A.P.; Hutch, W.; Borre, Y.E.; Kennedy, P.J.; Temko, A.; Boylan, G.; Murphy, E.; Cryan, J.F.; Dinan, T.G.; Clarke, G. Bifidobacterium longum 1714 as a translational psychobiotic: Modulation of stress, electrophysiology and neurocognition in healthy volunteers. Transl. Psychiatry 2016, 6, e939. [CrossRef]

35. Benton, D.; Williams, C.; Brown, A. Impact of consuming a milk drink containing a probiotic on mood and cognition. Eur. J. Clin. Nutr. 2007, 61, 355-361. [CrossRef]

36. Chung, Y.-C.; Jin, H.-M.; Cui, Y.; Kim, D.S.; Jung, J.M.; Park, J.-I.; Jung, E.-S.; Choi, E.-K.; Chae, S.-W. Fermented milk of Lactobacillus helveticus IDCC3801 improves cognitive functioning during cognitive fatigue tests in healthy older adults. J. Funct. Foods 2014, 10, 465-474. [CrossRef]

37. De Lorenzo, A.; Costacurta, M.; Merra, G.; Gualtieri, P.; Cioccoloni, G.; Marchetti, M.; Varvaras, D.; Docimo, R.; Di Renzo, L. Can psychobiotics intake modulate psychological profile and body composition of women affected by normal weight obese syndrome and obesity? A double blind randomized clinical trial. J. Transl. Med. 2017, 15, 135. [CrossRef]

38. Dickerson, F.B.; Stallings, C.; Origoni, A.; Katsafanas, E.; Savage, C.L.; Schweinfurth, L.A.; Goga, J.; Khushalani, S.; Yolken, R.H. Effect of probiotic supplementation on schizophrenia symptoms and association with gastrointestinal functioning: A randomized, placebo-controlled trial. Prim. Care Companion CNS Disord. 2014, 16, 26294. [CrossRef]

39. Dickerson, F.; Adamos, M.; Katsafanas, E.; Khushalani, S.; Origoni, A.; Savage, C.; Schweinfurth, L.; Stallings, C.; Sweeney, K.; Goga, J.; et al. Adjunctive probiotic microorganisms to prevent rehospitalization in patients with acute mania: A randomized controlled trial. Bipolar Disord. 2018, 20, 614-621. [CrossRef]

40. Diop, L.; Guillou, S.; Durand, H. Probiotic food supplement reduces stress-induced gastrointestinal symptoms in volunteers: A double-blind, placebo-controlled, randomized trial. Nutr. Res. 2008, 28, 1-5. [CrossRef]

41. Ghaderi, A.; Banafshe, H.R.; Mirhosseini, N.; Moradi, M.; Karimi, M.A.; Mehrzad, F.; Bahmani, F.; Asemi, Z. Clinical and metabolic response to vitamin D plus probiotic in schizophrenia patients. BMC Psychiatry 2019, 19, 77. [CrossRef]

42. Gualtieri, P.; Marchetti, M.; Cioccoloni, G.; De Lorenzo, A.; Romano, L.; Cammarano, A.; Colica, C.; Condò, R.; Di Renzo, L. Psychobiotics Regulate the Anxiety Symptoms in Carriers of Allele A of IL-1 $\beta$ Gene: A Randomized, Placebo-Controlled Clinical Trial. Mediat. Inflamm. 2020, 2020, 2346126. [CrossRef]

43. Ho, Y.T.; Tsai, Y.C.; Kuo, T.B.J.; Yang, C.C.H. Effects of Lactobacillus plantarum PS128 on Depressive Symptoms and Sleep Quality in Self-Reported Insomniacs: A Randomized, Double-Blind, Placebo-Controlled Pilot Trial. Nutrients 2021, 13, 2820. [CrossRef]

44. Hwang, Y.H.; Park, S.; Paik, J.W.; Chae, S.W.; Kim, D.H.; Jeong, D.G.; Ha, E.; Kim, M.; Hong, G.; Park, S.H.; et al. Efficacy and Safety of Lactobacillus Plantarum C29-Fermented Soybean (DW2009) in Individuals with Mild Cognitive Impairment: A 12-Week, Multi-Center, Randomized, Double-Blind, Placebo-Controlled Clinical Trial. Nutrients 2019, 11, 305. [CrossRef]

45. Inoue, T.; Kobayashi, Y.; Mori, N.; Sakagawa, M.; Xiao, J.Z.; Moritani, T.; Sakane, N.; Nagai, N. Effect of combined bifidobacteria supplementation and resistance training on cognitive function, body composition and bowel habits of healthy elderly subjects. Benef. Microbes 2018, 9, 843-853. [CrossRef]

46. Karbownik, M.S.; Kręczyńska, J.; Kwarta, P.; Cybula, M.; Wiktorowska-Owczarek, A.; Kowalczyk, E.; Pietras, T.; Szemraj, J. Effect of Supplementation with Saccharomyces Boulardii on Academic Examination Performance and Related Stress in Healthy Medical Students: A Randomized, Double-Blind, Placebo-Controlled Trial. Nutrients 2020, 12, 1469. [CrossRef]

47. Kato-Kataoka, A.; Nishida, K.; Takada, M.; Kawai, M.; Kikuchi-Hayakawa, H.; Suda, K.; Ishikawa, H.; Gondo, Y.; Shimizu, K.; Matsuki, T.; et al. Fermented Milk Containing Lactobacillus casei Strain Shirota Preserves the Diversity of the Gut Microbiota and Relieves Abdominal Dysfunction in Healthy Medical Students Exposed to Academic Stress. Appl. Environ. Microbiol. 2016, 82, 3649-3658. [CrossRef]

48. Kazemi, A.; Noorbala, A.A.; Azam, K.; Eskandari, M.H.; Djafarian, K. Effect of probiotic and prebiotic vs placebo on psychological outcomes in patients with major depressive disorder: A randomized clinical trial. Clin. Nutr. 2019, 38, 522-528. [CrossRef]

49. Kelly, J.R.; Allen, A.P.; Temko, A.; Hutch, W.; Kennedy, P.J.; Farid, N.; Murphy, E.; Boylan, G.; Bienenstock, J.; Cryan, J.F.; et al. Lost in translation? The potential psychobiotic Lactobacillus rhamnosus (JB-1) fails to modulate stress or cognitive performance in healthy male subjects. Brain Behav. Immun. 2017, 61, 50-59. [CrossRef]

50. Kobayashi, Y.; Kuhara, T.; Oki, M.; Xiao, J.Z. Effects of Bifidobacterium breve A1 on the cognitive function of older adults with memory complaints: A randomised, double-blind, placebo-controlled trial. Benef. Microbes 2019, 10, 511-520. [CrossRef] [PubMed]

51. Lew, L.C.; Hor, Y.Y.; Yusoff, N.A.A.; Choi, S.B.; Yusoff, M.S.B.; Roslan, N.S.; Ahmad, A.; Mohammad, J.A.M.; Abdullah, M.; Zakaria, N.; et al. Probiotic Lactobacillus plantarum P8 alleviated stress and anxiety while enhancing memory and cognition in stressed adults: A randomised, double-blind, placebo-controlled study. Clin. Nutr. 2019, 38, 2053-2064. [CrossRef] [PubMed]

52. Messaoudi, M.; Lalonde, R.; Violle, N.; Javelot, H.; Desor, D.; Nejdi, A.; Bisson, J.F.; Rougeot, C.; Pichelin, M.; Cazaubiel, M.; et al. Assessment of psychotropic-like properties of a probiotic formulation (Lactobacillus helveticus R0052 and Bifidobacterium longum R0175) in rats and human subjects. Br. J. Nutr. 2011, 105, 755-764. [CrossRef] [PubMed]

53. Mohammadi, A.A.; Jazayeri, S.; Khosravi-Darani, K.; Solati, Z.; Mohammadpour, N.; Asemi, Z.; Adab, Z.; Djalali, M.; TehraniDoost, M.; Hosseini, M.; et al. The effects of probiotics on mental health and hypothalamic-pituitary-adrenal axis: A randomized, double-blind, placebo-controlled trial in petrochemical workers. Nutr. Neurosci. 2016, 19, 387-395. [CrossRef] [PubMed]

54. Murata, M.; Kondo, J.; Iwabuchi, N.; Takahashi, S.; Yamauchi, K.; Abe, F.; Miura, K. Effects of paraprobiotic Lactobacillus paracasei MCC1849 supplementation on symptoms of the common cold and mood states in healthy adults. Benef. Microbes 2018, 9, 855-864. [CrossRef] [PubMed] 
55. Nishida, K.; Sawada, D.; Kawai, T.; Kuwano, Y.; Fujiwara, S.; Rokutan, K. Para-psychobiotic Lactobacillus gasseri CP2305 ameliorates stress-related symptoms and sleep quality. J. Appl. Microbiol. 2017, 123, 1561-1570. [CrossRef]

56. Nishida, K.; Sawada, D.; Kuwano, Y.; Tanaka, H.; Rokutan, K. Health Benefits of Lactobacillus gasseri CP2305 Tablets in Young Adults Exposed to Chronic Stress: A Randomized, Double-Blind, Placebo-Controlled Study. Nutrients 2019, 11, 1859. [CrossRef]

57. Nishihira, J.; Kagami-Katsuyama, H.; Tanaka, A.; Nishimura, M.; Kobayashi, T.; Kawasaki, Y. Elevation of natural killer cell activity and alleviation of mental stress by the consumption of yogurt containing Lactobacillus gasseri SBT2055 and Bifidobacterium longum SBT2928 in a double-blind, placebo-controlled clinical trial. J. Funct. Foods 2014, 11, 261-268. [CrossRef]

58. Ohsawa, K.; Nakamura, F.; Uchida, N.; Mizuno, S.; Yokogoshi, H. Lactobacillus helveticus-fermented milk containing lactononadecapeptide (NIPPLTQTPVVVPPFLQPE) improves cognitive function in healthy middle-aged adults: A randomised, double-blind, placebo-controlled trial. Int. J. Food Sci. Nutr. 2018, 69, 369-376. [CrossRef]

59. Östlund-Lagerström, L.; Kihlgren, A.; Repsilber, D.; Björkstén, B.; Brummer, R.J.; Schoultz, I. Probiotic administration among free-living older adults: A double blinded, randomized, placebo-controlled clinical trial. Nutr. J. 2016, 15, 80. [CrossRef]

60. Patterson, E.; Griffin, S.M.; Ibarra, A.; Ellsiepen, E.; Hellhammer, J. Lacticaseibacillus paracasei Lpc-37®improves psychological and physiological markers of stress and anxiety in healthy adults: A randomized, double-blind, placebo-controlled and parallel clinical trial (the Sisu study). Neurobiol. Stress 2020, 13, 100277. [CrossRef]

61. Rao, A.V.; Bested, A.C.; Beaulne, T.M.; Katzman, M.A.; Iorio, C.; Berardi, J.M.; Logan, A.C. A randomized, double-blind, placebo-controlled pilot study of a probiotic in emotional symptoms of chronic fatigue syndrome. Gut Pathog. 2009, 1, 6. [CrossRef]

62. Raygan, F.; Ostadmohammadi, V.; Bahmani, F.; Asemi, Z. The effects of vitamin D and probiotic co-supplementation on mental health parameters and metabolic status in type 2 diabetic patients with coronary heart disease: A randomized, double-blind, placebo-controlled trial. Prog. Neuropsychopharmacol. Biol. Psychiatry 2018, 84, 50-55. [CrossRef]

63. Raygan, F.; Ostadmohammadi, V.; Asemi, Z. The effects of probiotic and selenium co-supplementation on mental health parameters and metabolic profiles in type 2 diabetic patients with coronary heart disease: A randomized, double-blind, placebocontrolled trial. Clin. Nutr. 2019, 38, 1594-1598. [CrossRef]

64. Reininghaus, E.Z.; Platzer, M.; Kohlhammer-Dohr, A.; Hamm, C.; Mörkl, S.; Bengesser, S.A.; Fellendorf, F.T.; LahousenLuxenberger, T.; Leitner-Afschar, B.; Schöggl, H.; et al. PROVIT: Supplementary Probiotic Treatment and Vitamin B7 in Depression-A Randomized Controlled Trial. Nutrients 2020, 12, 3422. [CrossRef]

65. Roman, P.; Estévez, A.F.; Miras, A.; Sánchez-Labraca, N.; Cañadas, F.; Vivas, A.B.; Cardona, D. A Pilot Randomized Controlled Trial to Explore Cognitive and Emotional Effects of Probiotics in Fibromyalgia. Sci. Rep. 2018, 8, 10965. [CrossRef]

66. Romijn, A.R.; Rucklidge, J.J.; Kuijer, R.G.; Frampton, C. A double-blind, randomized, placebo-controlled trial of Lactobacillus helveticus and Bifidobacterium longum for the symptoms of depression. Aust. N. Z. J. Psychiatry 2017, 51, 810-821. [CrossRef]

67. Rudzki, L.; Ostrowska, L.; Pawlak, D.; Małus, A.; Pawlak, K.; Waszkiewicz, N.; Szulc, A. Probiotic Lactobacillus Plantarum $299 \mathrm{v}$ decreases kynurenine concentration and improves cognitive functions in patients with major depression: A double-blind, randomized, placebo controlled study. Psychoneuroendocrinology 2019, 100, 213-222. [CrossRef]

68. Sanchez, M.; Darimont, C.; Panahi, S.; Drapeau, V.; Marette, A.; Taylor, V.H.; Doré, J.; Tremblay, A. Effects of a Diet-Based Weight-Reducing Program with Probiotic Supplementation on Satiety Efficiency, Eating Behaviour Traits, and Psychosocial Behaviours in Obese Individuals. Nutrients 2017, 9, 284. [CrossRef]

69. Sashihara, T.; Nagata, M.; Mori, T.; Ikegami, S.; Gotoh, M.; Okubo, K.; Uchida, M.; Itoh, H. Effects of Lactobacillus gasseri OLL2809 and $\alpha$-lactalbumin on university-student athletes: A randomized, double-blind, placebo-controlled clinical trial. Appl. Physiol. Nutr. Metab. 2013, 38, 1228-1235. [CrossRef]

70. Sawada, D.; Kawai, T.; Nishida, K.; Kuwano, Y.; Fujiwara, S.; Rokutan, K. Daily intake of Lactobacillus gasseri CP2305 improves mental, physical, and sleep quality among Japanese medical students enrolled in a cadaver dissection course. J. Funct. Foods 2017, 31, 188-197. [CrossRef]

71. Severance, E.G.; Gressitt, K.L.; Stallings, C.R.; Katsafanas, E.; Schweinfurth, L.A.; Savage, C.L.G.; Adamos, M.B.; Sweeney, K.M.; Origoni, A.E.; Khushalani, S.; et al. Probiotic normalization of Candida albicans in schizophrenia: A randomized, placebo-controlled, longitudinal pilot study. Brain Behav. Immun. 2017, 62, 41-45. [CrossRef]

72. Shinkai, S.; Toba, M.; Saito, T.; Sato, I.; Tsubouchi, M.; Taira, K.; Kakumoto, K.; Inamatsu, T.; Yoshida, H.; Fujiwara, Y.; et al. Immunoprotective effects of oral intake of heat-killed Lactobacillus pentosus strain b240 in elderly adults: A randomised, double-blind, placebo-controlled trial. Br. J. Nutr. 2013, 109, 1856-1865. [CrossRef]

73. Steenbergen, L.; Sellaro, R.; van Hemert, S.; Bosch, J.A.; Colzato, L.S. A randomized controlled trial to test the effect of multispecies probiotics on cognitive reactivity to sad mood. Brain Behav. Immun. 2015, 48, 258-264. [CrossRef]

74. Takada, M.; Nishida, K.; Kataoka-Kato, A.; Gondo, Y.; Ishikawa, H.; Suda, K.; Kawai, M.; Hoshi, R.; Watanabe, O.; Igarashi, T.; et al. Probiotic Lactobacillus casei strain Shirota relieves stress-associated symptoms by modulating the gut-brain interaction in human and animal models. Neurogastroenterol. Motil. 2016, 28, 1027-1036. [CrossRef]

75. Takada, M.; Nishida, K.; Gondo, Y.; Kikuchi-Hayakawa, H.; Ishikawa, H.; Suda, K.; Kawai, M.; Hoshi, R.; Kuwano, Y.; Miyazaki, K.; et al. Beneficial effects of Lactobacillus casei strain Shirota on academic stress-induced sleep disturbance in healthy adults: A double-blind, randomised, placebo-controlled trial. Benef. Microbes 2017, 8, 153-162. [CrossRef] 
76. Tran, N.; Zhebrak, M.; Yacoub, C.; Pelletier, J.; Hawley, D. The gut-brain relationship: Investigating the effect of multispecies probiotics on anxiety in a randomized placebo-controlled trial of healthy young adults. J. Affect. Disord. 2019, 252, $271-277$. [CrossRef]

77. Xiao, J.; Katsumata, N.; Bernier, F.; Ohno, K.; Yamauchi, Y.; Odamaki, T.; Yoshikawa, K.; Ito, K.; Kaneko, T. Probiotic Bifidobacterium breve in Improving Cognitive Functions of Older Adults with Suspected Mild Cognitive Impairment: A Randomized, Double-Blind, Placebo-Controlled Trial. J. Alzheimers Dis. 2020, 77, 139-147. [CrossRef]

78. Yamamura, S.; Morishima, H.; Kumano-go, T.; Suganuma, N.; Matsumoto, H.; Adachi, H.; Sigedo, Y.; Mikami, A.; Kai, T.; Masuyama, A.; et al. The effect of Lactobacillus helveticus fermented milk on sleep and health perception in elderly subjects. Eur. J. Clin. Nutr. 2009, 63, 100-105. [CrossRef]

79. Zhang, X.; Chen, S.; Zhang, M.; Ren, F.; Ren, Y.; Li, Y.; Liu, N.; Zhang, Y.; Zhang, Q.; Wang, R. Effects of Fermented Milk Containing Lacticaseibacillus paracasei Strain Shirota on Constipation in Patients with Depression: A Randomized, Double-Blind, Placebo-Controlled Trial. Nutrients 2021, 13, 2238. [CrossRef]

80. Preidis, G.A.; Weizman, A.V.; Kashyap, P.C.; Morgan, R.L. AGA Technical Review on the Role of Probiotics in the Management of Gastrointestinal Disorders. Gastroenterology 2020, 159, 708-738.E4. [CrossRef]

81. Mazurak, N.; Broelz, E.; Storr, M.; Enck, P. Probiotic Therapy of the Irritable Bowel Syndrome: Why Is the Evidence Still Poor and What Can Be Done About It? J. Neurogastroenterol. Motil. 2015, 21, 471-485. [CrossRef]

82. O'Toole, P.W.; Marchesi, J.R.; Hill, C. Next-generation probiotics: The spectrum from probiotics to live biotherapeutics. Nat. Microbiol. 2017, 2, 17057. [CrossRef] [PubMed] 